\title{
Inclusive-jet photoproduction at HERA and determination of $\alpha_{s}$
}

\section{ZEUS Collaboration}

H. Abramowicz ${ }^{\text {as,53 }}$, I. Abt ${ }^{\text {ai }}$, L. Adamczyk ${ }^{\mathrm{m}}$, M. Adamus ${ }^{\mathrm{bb}}$, R. Aggarwal ${ }^{\mathrm{g}, 21}, \mathrm{~S}$. Antonelli ${ }^{\mathrm{d}}$, P. Antonioli ${ }^{\mathrm{c}}$, A. Antonov ${ }^{\mathrm{ag}}$, M. Arneodo ax , V. Aushev ${ }^{\text {z,aa,45 }}$, Y. Aushev ${ }^{\text {aa, } 45,46}$, O. Bachynska ${ }^{\circ}$, A. Bamberger ${ }^{\mathrm{s}}$, A.N. Barakbaev ${ }^{\mathrm{y}}$, G. Barbagli ${ }^{\mathrm{q}}$, G. Bari ${ }^{\mathrm{c}}$, F. Barreiro ${ }^{\mathrm{ad}}$, N. Bartosik ${ }^{\circ}$, D. Bartsch ${ }^{\mathrm{e}}, \mathrm{M}$. Basile $^{\mathrm{d}}, \mathrm{O}$. Behnke $^{\mathrm{o}}, \mathrm{J}_{\text {. Behr }}{ }^{\text {, }}$, U. Behrens $^{\circ}$, L. Bellagamba ${ }^{\text {c }}$, A. Bertolin ${ }^{\text {am }}$, S. Bhadra ${ }^{\text {be }}$, M. Bindi ${ }^{\text {d, }}$ C. Blohm ${ }^{\mathrm{o}}$, V. Bokhonov ${ }^{\mathrm{z}, 45}$, T. Bołd ${ }^{\mathrm{m}}$, K. Bondarenko ${ }^{\text {aa }}$, E.G. Boos ${ }^{\mathrm{y}}$, K. Borras ${ }^{\mathrm{o}}$, D. Boscherini ${ }^{\mathrm{c}}$, D. Bot ${ }^{\mathrm{o}}, \mathrm{I}_{\text {. Brock }}^{\mathrm{e}}$, E. Brownson $^{\mathrm{bd}}$, R. Brugnera ${ }^{\text {an }}$, N. Brümmer ${ }^{\text {ak }}$, A. Bruni ${ }^{c}$, G. Bruni ${ }^{c}$, B. Brzozowska ${ }^{\text {ba }}$, P.J. Bussey ${ }^{\mathrm{t}}$, B. Bylsma ${ }^{\text {ak }}$, A. Caldwell ${ }^{\text {ai }}$, M. Capua ${ }^{\text {h }}$, R. Carlin ${ }^{\text {an }}$, C.D. Catterall ${ }^{\text {be }}$, S. Chekanov ${ }^{\text {a }}$, J. Chwastowski ${ }^{1,23}$, J. Ciborowski ${ }^{\text {ba,57, }}$, R. Ciesielski ${ }^{\mathrm{o}, 26}$, L. Cifarelli ${ }^{\mathrm{d}}$, F. Cindolo ${ }^{\mathrm{c}}$, A. Contin $^{\mathrm{d}}$, A.M. Cooper-Sarkar ${ }^{\text {al }}$, N. Coppola ${ }^{\mathrm{o}, 27}$, M. Corradi ${ }^{\mathrm{c}}$, F. Corriveau ${ }^{\mathrm{ae}}$, M. Costa ${ }^{\text {aw }}$, G. D’Agostini ${ }^{\text {aq }}$, F. Dal Corso ${ }^{\text {am }}$, J. del Peso ${ }^{\text {ad }}$, R.K. Dementiev ah, S. De Pasquale ${ }^{\text {d,19 }}{ }^{\text {, M. Derrick }}{ }^{\text {a }}$, R.C.E. Devenish ${ }^{\text {al }}$, D. Dobur ${ }^{\mathrm{s}, 38}$, B.A. Dolgoshein ${ }^{\mathrm{ag}, 61}$, G. Dolinska ${ }^{\text {aa }}$, A.T. Doyle ${ }^{\mathrm{t}}$, V. Drugakov ${ }^{\mathrm{p}}$, L.S. Durkin ${ }^{\text {ak }}$, S. Dusini ${ }^{\mathrm{am}}$, Y. Eisenberg ${ }^{\mathrm{bc}}$, P.F. Ermolov ${ }^{\text {ah,61 }}$, A. Eskreys ${ }^{1,61}$, S. Fang ${ }^{\mathrm{o}, 28}$, S. Fazio ${ }^{\text {h }}$, J. Ferrando ${ }^{\text {al }}$, M.I. Ferrero ${ }^{\text {aw }}$, J. Figiel ${ }^{1}$, M. Forrest ${ }^{\mathrm{t}, 41}$, B. Foster ${ }^{\mathrm{al}, 49}$, G. Gach ${ }^{\mathrm{m}}$, A. Galas ${ }^{1}$, E. Gallo ${ }^{\mathrm{y}}$, A. Garfagnini ${ }^{\text {an }}$, A. Geiser ${ }^{\circ}$, I. Gialas $^{\mathrm{u}, 42}$, A. Gizhko ${ }^{\text {aa,47 }}$, L.K. Gladilin ${ }^{\text {ah,48, D. Gladkov }}{ }^{\text {ag }}$, C. Glasman ${ }^{\text {ad }}$, O. Gogota $^{\text {aa }}$, Yu.A. Golubkov ${ }^{\text {ah }}$, P. Göttlicher ${ }^{0,29}$, I. Grabowska-Bołd ${ }^{\text {m }}$, J. Grebenyuk ${ }^{\text {, }}$ I. Gregor ${ }^{\circ}$, G. Grigorescu ${ }^{\text {aj }}$, G. Grzelak ${ }^{\text {ba }}$, O. Gueta ${ }^{\text {as }}$, M. Guzik ${ }^{\mathrm{m}}$, C. Gwenlan ${ }^{\mathrm{al}, 50}$, T. Haas $^{\circ}$, W. Hain ${ }^{\circ}$, R. Hamatsu ${ }^{\text {av }}$, J.C. Hart $^{\text {ar }}$, H. Hartmann ${ }^{\text {e }}$, G. Hartner ${ }^{\text {be }}$, E. Hilger ${ }^{\text {e }}$, D. Hochman ${ }^{\text {bc }}$, 
R. Hori ${ }^{\text {au }}$, K. Horton ${ }^{\text {al,51 }}$, A. Hüttmann ${ }^{\text {, }}$, Z.A. Ibrahim ${ }^{\mathrm{j}}$, Y. Iga ${ }^{\text {ap }}$, R. Ingbir ${ }^{\text {as }}$, M. Ishitsuka ${ }^{\text {at }}$, H.-P. Jakob ${ }^{\mathrm{e}}$, F. Januschek ${ }^{\mathrm{o}}$, T.W. Jones ${ }^{\text {az }}$, M. Jüngst ${ }^{\mathrm{e}}$, I. Kadenko ${ }^{\text {aa }}$, B. Kahle ${ }^{\mathrm{o}}$, S. Kananov ${ }^{\text {as }}$, T. Kanno ${ }^{\text {at }}$, U. Karshon ${ }^{\text {bc }}$, F. Karstens ${ }^{\text {s,39}}$, I.I. Katkov ${ }^{\text {o, } 30}$, M. Kaur ${ }^{\mathrm{g}}$, P. Kaur ${ }^{\mathrm{g}, 21}$,

A. Keramidas ${ }^{\text {aj }}$, L.A. Khein ${ }^{\text {ah }}$, J.Y. Kim ${ }^{\text {i }}$, D. Kisielewska ${ }^{\mathrm{m}}$, S. Kitamura ${ }^{\text {av,55, }}$, R. Klanner ${ }^{\text {v }}$, U. Klein ${ }^{\text {o,31, E. Koffeman }}{ }^{\text {aj, }}$, N. Kondrashova a ${ }^{\text {aa }} 47$, O. Kononeko ${ }^{\text {aa }}$, P. Kooijman ${ }^{\text {aj }}$, Ie. Korol ${ }^{\text {aa }}$, I.A. Korzhavina $^{\text {ah,48 }}$, A. Kotański ${ }^{\text {n,24 }}$, U. Kötz ${ }^{\text {o }}$, H. Kowalski ${ }^{\text {, }}$, O. Kuprash ${ }^{\circ}$, M. Kuze ${ }^{\text {at }}$, A. Lee ${ }^{\text {ak }}$, B.B. Levchenko ${ }^{\text {ah }}$, A. Levy ${ }^{\text {as,*, }}$, V. Libov ${ }^{\circ}$, S. Limentani ${ }^{\text {an }}$, T.Y. Ling ${ }^{\text {ak }}$, M. Lisovyi ${ }^{\circ}$, E. Lobodzinska $^{\circ}$, W. Lohmann ${ }^{\mathrm{p}}$, B. Löhr ${ }^{\mathrm{o}}$, E. Lohrmann ${ }^{\mathrm{v}}$, K.R. Long ${ }^{\mathrm{w}}$, A. Longhin ${ }^{\mathrm{am}}$,

D. Lontkovskyi ${ }^{\circ}$, O.Yu. Lukina ${ }^{\text {ah }}$, J. Maeda $^{\text {at,54 }}$, S. Magill ${ }^{\text {a }}$, I. Makarenko ${ }^{\circ}$, J. Malka $^{\circ}$, R. Mankel ${ }^{\circ}$, A. Margotti ${ }^{\text {c }}$, G. Marini ${ }^{\text {aq }}$, J.F. Martin ay , A. Mastroberardino ${ }^{\text {h }}$, M.C.K. Mattingly ${ }^{\mathrm{b}}$,

I.-A. Melzer-Pellmann $^{\circ}$, S. Mergelmeyer ${ }^{\mathrm{e}}, \mathrm{S}$. Miglioranzi ${ }^{\mathrm{o}, 32}$, F. Mohamad Idris ${ }^{j}$, V. Monaco ${ }^{\text {aw }}$, A. Montanari ${ }^{\circ}$, J.D. Morris ${ }^{\text {f,20, }}$, K. Mujkic ${ }^{0,33}$, B. Musgrave ${ }^{\text {a }}$, K. Nagano ${ }^{\mathrm{x}}$, T. Namsoo ${ }^{\mathrm{o}, 34}, \mathrm{~N}_{\text {. Nania }}{ }^{\mathrm{c}}$, A. Nigro ${ }^{\text {aq }}$, Y. Ning ${ }^{\mathrm{k}}$, T. Nobe ${ }^{\text {at }}$, U. Noor ${ }^{\text {be }}$, D. Notz ${ }^{\circ}$, R.J. Nowak ${ }^{\text {ba }}$, A.E. Nuncio-Quiroz ${ }^{\mathrm{e}}$, B.Y. Oh ${ }^{\mathrm{ao}}$, N. Okazaki ${ }^{\text {au }}$, K. Oliver ${ }^{\mathrm{al}}$, K. Olkiewicz ${ }^{1}$, Yu. Onishchuk ${ }^{\text {aa }}$, K. Papageorgiu ${ }^{\text {u }}$, A. Parenti ${ }^{\circ}$, E. Paul ${ }^{\text {e }}$, J.M. Pawlak ${ }^{\text {ba }}$, B. Pawlik ${ }^{1}$, P.G. Pelfer ${ }^{\mathrm{r}}$, A. Pellegrino ${ }^{\text {aj }}$, W. Perlański ${ }^{\text {ba,58, }}{ }^{\text {, }}$ H. Perrey ${ }^{o}$, K. Piotrzkowski ${ }^{\text {ac }}$, P. Pluciński ${ }^{\text {bb,59 }}{ }^{\text {, N.S. Pokrovskiy }}{ }^{\mathrm{y}}$,

A. Polini ${ }^{\mathrm{c}}$, A.S. Proskuryakov ${ }^{\text {ah }}$, M. Przybycień ${ }^{\mathrm{m}}$, A. Raval ${ }^{\circ}$, D.D. Reeder ${ }^{\text {bd }}$, B. Reisert ${ }^{\text {ai }}$, Z. Ren ${ }^{\text {k }}$, J. Repond ${ }^{\text {a }}$, Y.D. Ri ${ }^{\text {av, } 56}$, A. Robertson ${ }^{\text {al }}$, P. Roloff ${ }^{o, 32}$, I. Rubinsky ${ }^{\circ}$, M. Ruspa ${ }^{\text {ax }}$, R. Sacchi ${ }^{\text {aw }}$, U. Samson ${ }^{\mathrm{e}}$, G. Sartorelli ${ }^{\mathrm{d}}$, A.A. Savin ${ }^{\text {bd }}$, D.H. Saxon ${ }^{\mathrm{t}}$, M. Schioppa ${ }^{\mathrm{h}}$, S. Schlenstedt $^{p}$, P. Schleper ${ }^{v}$, W.B. Schmidke ${ }^{\text {ai }}$, U. Schneekloth ${ }^{\circ}$,

V. Schönberg e, T. Schörner-Sadenius ${ }^{\circ}$, J. Schwartz ${ }^{\text {ae }}$, F. Sciulli ${ }^{\text {k, }}$, L.M. Shcheglova ${ }^{\text {ah }}$, R. Shehzadi ${ }^{\text {e }}$, S. Shimizu ${ }^{\text {au, } 32}$, I. Singh ${ }^{\text {g, } 21}$, I.O. Skillicorn ${ }^{\mathrm{t}}$, W. Słomiński ${ }^{\mathrm{n}, 25}$, W.H. Smith ${ }^{\text {bd }}$, V. Sola ${ }^{\text {aw }}$, A. Solano ${ }^{\text {aw }}$, D. Son ${ }^{\mathrm{ab}}$, V. Sosnovtsev ag ${ }^{\mathrm{a}}$ A. Spiridonov ${ }^{0,35}$, H. Stadie ${ }^{\mathrm{v}}$, L. Stanco ${ }^{\mathrm{am}}$, N. Stefaniuk ${ }^{\text {aa }}$, A. Stern ${ }^{\text {as }}$, T.P. Stewart ${ }^{\text {ay }}$, A. Stifutkin ${ }^{\text {ag }}$, P. Stopa ${ }^{1}$,

S. Suchkov ${ }^{\text {ag }}$, G. Susinno ${ }^{\text {h }}$, L. Suszycki ${ }^{\mathrm{m}}$, J. Sztuk-Dambietz ${ }^{\mathrm{v}}$, D. Szuba ${ }^{\text {}}$, J. Szuba ${ }^{0,36}$, A.D. Tapper ${ }^{\mathrm{w}}$, E. Tassi ${ }^{\text {h,22 }}$, J. Terrón ${ }^{\text {ad }}$, T. Theedt ${ }^{\circ}$, H. Tiecke ${ }^{\text {aj }}$, K. Tokushuku ${ }^{\mathrm{x}, 43}$, J. Tomaszewska $^{0,37}$, V. Trusov ${ }^{\text {aa }}$, T. Tsurugai ${ }^{\text {af }}$, M. Turcato ${ }^{\mathrm{v}}$, O. Turkot $^{\text {aa, } 47}$, T. Tymieniecka ${ }^{\text {bb,60 }}$, M. Vázquez ${ }^{\text {aj, } 32}$, A. Verbytskyi ${ }^{\circ}$, O. Viazlo ${ }^{\text {aa }}$, 


\title{
N.N. Vlasov ${ }^{\mathrm{s}, 40}$, R. Walczak ${ }^{\text {al }}$, W.A.T. Wan Abdullah ${ }^{\mathrm{j}}$, J.J. Whitmore ${ }^{\text {ao, } 52}$, L. Wiggers ${ }^{\text {aj }}$, M. Wing ${ }^{\text {az }}$, M. Wlasenko ${ }^{\mathrm{e}}$, G. Wolf ${ }^{\circ}$, H. Wolfe ${ }^{\text {bd }}$, K. Wrona ${ }^{\circ}$, A.G. Yagües-Molina ${ }^{\circ}$, S. Yamada ${ }^{\mathrm{x}}$, Y. Yamazaki ${ }^{\mathrm{x}, 44}$, R. Yoshida ${ }^{\mathrm{a}}$, C. Youngman ${ }^{\circ}$, O. Zabiegalov $^{\mathrm{aa}, 47}$, A.F. Żarnecki ${ }^{\text {ba }}$, L. Zawiejski ${ }^{1}$, O. Zenaiev ${ }^{\circ}$, W. Zeuner ${ }^{0,32}$, B.O. Zhautykov ${ }^{\mathrm{y}}$, N. Zhmak ${ }^{\text {z,45 }}$, C. Zhou ${ }^{\text {ae }}$, A. Zichichi ${ }^{\mathrm{d}}$, Z. Zolkapli ${ }^{\mathrm{j}}$, D.S. Zotkin ah
}

\author{
a Argonne National Laboratory, Argonne, IL 60439-4815, USA ${ }^{1}$ \\ b Andrews University, Berrien Springs, MI 49104-0380, USA \\ c INFN Bologna, Bologna, Italy ${ }^{2}$ \\ ${ }^{\mathrm{d}}$ University and INFN Bologna, Bologna, Italy ${ }^{2}$ \\ e Physikalisches Institut der Universität Bonn, Bonn, Germany ${ }^{3}$ \\ ${ }^{\mathrm{f}}$ H.H. Wills Physics Laboratory, University of Bristol, Bristol, United Kingdom ${ }^{4}$ \\ g Panjab University, Department of Physics, Chandigarh, India \\ h Calabria University, Physics Department and INFN, Cosenza, Italy ${ }^{2}$ \\ ${ }^{\mathrm{i}}$ Institute for Universe and Elementary Particles, Chonnam National University, Kwangju, South Korea \\ j Jabatan Fizik, Universiti Malaya, 50603 Kuala Lumpur, Malaysia ${ }^{5}$ \\ ${ }^{\mathrm{k}}$ Nevis Laboratories, Columbia University, Irvington on Hudson, NY 10027, USA 6 \\ ${ }^{1}$ The Henryk Niewodniczanski Institute of Nuclear Physics, Polish Academy of Sciences, Krakow, Poland ${ }^{7}$ \\ ${ }^{\mathrm{m}}$ AGH-University of Science and Technology, Faculty of Physics and Applied Computer Science, Krakow, Poland ${ }^{8}$ \\ ${ }^{\mathrm{n}}$ Department of Physics, Jagellonian University, Cracow, Poland \\ o Deutsches Elektronen-Synchrotron DESY, Hamburg, Germany \\ p Deutsches Elektronen-Synchrotron DESY, Zeuthen, Germany \\ q INFN Florence, Florence, Italy ${ }^{2}$ \\ ${ }^{\mathrm{r}}$ University and INFN Florence, Florence, Italy ${ }^{2}$ \\ ${ }^{\mathrm{s}}$ Fakultät für Physik der Universität Freiburg i.Br., Freiburg i.Br., Germany \\ ${ }^{\mathrm{t}}$ School of Physics and Astronomy, University of Glasgow, Glasgow, United Kingdom ${ }^{4}$ \\ ${ }^{\mathrm{u}}$ Department of Engineering in Management and Finance, Univ. of the Aegean, Chios, Greece \\ ${ }^{\mathrm{v}}$ Hamburg University, Institute of Experimental Physics, Hamburg, Germany ${ }^{9}$ \\ ${ }^{w}$ Imperial College London, High Energy Nuclear Physics Group, London, United Kingdom 4 \\ ${ }^{\mathrm{x}}$ Institute of Particle and Nuclear Studies, KEK, Tsukuba, Japan ${ }^{10}$ \\ ${ }^{\mathrm{y}}$ Institute of Physics and Technology of Ministry of Education and Science of Kazakhstan, Almaty, Kazakhstan \\ ${ }^{\mathrm{z}}$ Institute for Nuclear Research, National Academy of Sciences, Kyiv, Ukraine \\ aa Department of Nuclear Physics, National Taras Shevchenko University of Kyiv, Kyiv, Ukraine \\ ab Kyungpook National University, Center for High Energy Physics, Daegu, South Korea 11 \\ ${ }^{a c}$ Institut de Physique Nucléaire, Université Catholique de Louvain, Louvain-la-Neuve, Belgium 12

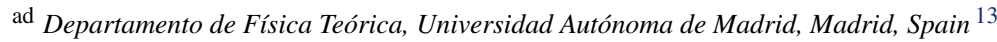 \\ ae Department of Physics, McGill University, Montréal, Québec, Canada H3A 2T8 ${ }^{14}$

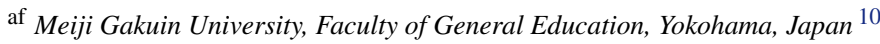 \\ ag Moscow Engineering Physics Institute, Moscow, Russia 15 \\ ah Lomonosov Moscow State University, Skobeltsyn Institute of Nuclear Physics, Moscow, Russia 16 \\ ai Max-Planck-Institut für Physik, München, Germany \\ aj NIKHEF and University of Amsterdam, Amsterdam, The Netherlands 17 \\ ${ }^{\text {ak }}$ Physics Department, Ohio State University, Columbus, OH 43210, USA ${ }^{1}$

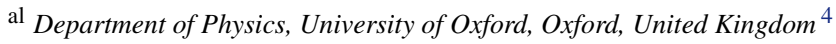 \\ am INFN Padova, Padova, Italy ${ }^{2}$ \\ an Dipartimento di Fisica dell'Università and INFN, Padova, Italy ${ }^{2}$
}




\section{RAPID COMMUNICATION}

${ }^{\text {ao }}$ Department of Physics, Pennsylvania State University, University Park, Pennsylvania 16802, USA ${ }^{6}$

ap Polytechnic University, Sagamihara, Japan ${ }^{10}$

aq Dipartimento di Fisica, Università 'La Sapienza' and INFN, Rome, Italy ${ }^{2}$

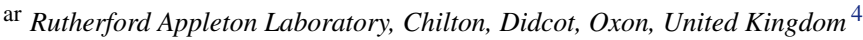

as Raymond and Beverly Sackler Faculty of Exact Sciences, School of Physics, Tel Aviv University, Tel Aviv, Israel ${ }^{18}$

at Department of Physics, Tokyo Institute of Technology, Tokyo, Japan ${ }^{10}$

au Department of Physics, University of Tokyo, Tokyo, Japan ${ }^{10}$

${ }^{\text {av }}$ Tokyo Metropolitan University, Department of Physics, Tokyo, Japan ${ }^{10}$

${ }^{\text {aw }}$ Università di Torino and INFN, Torino, Italy ${ }^{2}$

ax Università del Piemonte Orientale, Novara, and INFN, Torino, Italy ${ }^{2}$

ay Department of Physics, University of Toronto, Toronto, Ontario, Canada M5S $1 A 7^{14}$

${ }^{\text {az }}$ Physics and Astronomy Department, University College London, London, United Kingdom ${ }^{4}$

ba Faculty of Physics, University of Warsaw, Warsaw, Poland

bb National Centre for Nuclear Research, Warsaw, Poland

bc Department of Particle Physics and Astrophysics, Weizmann Institute, Rehovot, Israel

bd Department of Physics, University of Wisconsin, Madison, WI 53706, USA ${ }^{1}$

be Department of Physics, York University, Ontario, Canada M3J 1P3 ${ }^{14}$

Received 4 June 2012; accepted 15 June 2012

Available online 22 June 2012

* Corresponding author.

E-mail address: levy@alzt.tau.ac.il (A. Levy).

1 Supported by the US Department of Energy.

2 Supported by the Italian National Institute for Nuclear Physics (INFN).

3 Supported by the German Federal Ministry for Education and Research (BMBF), under contract No. 05 H09PDF.

4 Supported by the Science and Technology Facilities Council, UK.

5 Supported by an FRGS grant from the Malaysian government.

6 Supported by the US National Science Foundation. Any opinion, findings and conclusions or recommendations expressed in this material are those of the authors and do not necessarily reflect the views of the National Science Foundation.

7 Supported by the Polish Ministry of Science and Higher Education as a scientific project No. DPN/N188/DESY/2009.

8 Supported by the Polish Ministry of Science and Higher Education and its grants for Scientific Research.

9 Supported by the German Federal Ministry for Education and Research (BMBF), under contract No. 05h09GUF, and the SFB 676 of the Deutsche Forschungsgemeinschaft (DFG).

10 Supported by the Japanese Ministry of Education, Culture, Sports, Science and Technology (MEXT) and its grants for Scientific Research.

11 Supported by the Korean Ministry of Education and Korea Science and Engineering Foundation.

12 Supported by FNRS and its associated funds (IISN and FRIA) and by an Inter-University Attraction Poles Programme subsidised by the Belgian Federal Science Policy Office.

13 Supported by the Spanish Ministry of Education and Science through funds provided by CICYT.

14 Supported by the Natural Sciences and Engineering Research Council of Canada (NSERC).

15 Partially supported by the German Federal Ministry for Education and Research (BMBF).

16 Supported by RF Presidential grant No. 4142.2010.2 for Leading Scientific Schools, by the Russian Ministry of Education and Science through its grant for Scientific Research on High Energy Physics and under contract No. 02.740.11.0244.

17 Supported by the Netherlands Foundation for Research on Matter (FOM).

18 Supported by the Israel Science Foundation.

19 Now at University of Salerno, Italy.

20 Now at Queen Mary University of London, United Kingdom. 


\begin{abstract}
Inclusive-jet cross sections have been measured in the reaction $e p \rightarrow e+$ jet $+\mathrm{X}$ for photon virtuality $Q^{2}<1 \mathrm{GeV}^{2}$ and $\gamma p$ centre-of-mass energies in the region $142<W_{\gamma p}<293 \mathrm{GeV}$ with the ZEUS detector at HERA using an integrated luminosity of $300 \mathrm{pb}^{-1}$. Jets were identified using the $k_{T}$, anti- $k_{T}$ or SIScone jet algorithms in the laboratory frame. Single-differential cross sections are presented as functions of the jet transverse energy, $E_{T}^{\text {jet }}$, and pseudorapidity, $\eta^{\text {jet }}$, for jets with $E_{T}^{\text {jet }}>17 \mathrm{GeV}$ and $-1<\eta^{\text {jet }}<2.5$. In addition, measurements of double-differential inclusive-jet cross sections are presented as functions of $E_{T}^{\text {jet }}$ in different regions of $\eta^{\text {jet }}$. Next-to-leading-order QCD calculations give a good description of the measurements, except for jets with low $E_{T}^{\text {jet }}$ and high $\eta^{\text {jet }}$. The influence of non-perturbative effects not related to hadronisation was studied. Measurements of the ratios of cross sections using different jet algorithms are also presented; the measured ratios are well described by calculations including up to $\mathcal{O}\left(\alpha_{s}^{2}\right)$ terms. Values of $\alpha_{S}\left(M_{Z}\right)$ were extracted from the measurements and the energy-scale dependence of the coupling was determined. The value of $\alpha_{S}\left(M_{Z}\right)$ extracted from the measurements based on the $k_{T}$ jet algorithm is $\alpha_{S}\left(M_{Z}\right)=0.1206_{-0.0022}^{+0.0023}$ (exp. $)_{-0.0035}^{+0.0042}$ (th.); the results from the anti- $k_{T}$ and SIScone algorithms are compatible with this value and have a similar precision.
\end{abstract}

(c) 2012 Elsevier B.V. All rights reserved.

21 Also funded by Max Planck Institute for Physics, Munich, Germany.

22 Also Senior Alexander von Humboldt Research Fellow at Hamburg University, Institute of Experimental Physics, Hamburg, Germany.

23 Also at Cracow University of Technology, Faculty of Physics, Mathematics and Applied Computer Science, Poland.

24 Supported by the research grant No. 1 P03B 04529 (2005-2008).

25 Supported by the Polish National Science Centre, project No. DEC-2011/01/BST2/03643.

26 Now at Rockefeller University, New York, NY 10065, USA.

27 Now at DESY group FS-CFEL-1.

28 Now at Institute of High Energy Physics, Beijing, China.

29 Now at DESY group FEB, Hamburg, Germany.

30 Also at Moscow State University, Russia.

31 Now at University of Liverpool, United Kingdom.

32 Now at CERN, Geneva, Switzerland.

33 Also affiliated with University College London, UK.

34 Now at Goldman Sachs, London, UK.

35 Also at Institute of Theoretical and Experimental Physics, Moscow, Russia.

36 Also at FPACS, AGH-UST, Cracow, Poland.

37 Partially supported by Warsaw University, Poland.

38 Now at Istituto Nucleare di Fisica Nazionale (INFN), Pisa, Italy.

39 Now at Haase Energie Technik AG, Neumünster, Germany.

40 Now at Department of Physics, University of Bonn, Germany.

41 Now at Biodiversität und Klimaforschungszentrum (BiK-F), Frankfurt, Germany.

42 Also affiliated with DESY, Germany.

43 Also at University of Tokyo, Japan.

44 Now at Kobe University, Japan.

45 Supported by DESY, Germany.

46 Member of National Technical University of Ukraine, Kyiv Polytechnic Institute, Kyiv, Ukraine.

47 Member of National University of Kyiv, Mohyla Academy, Kyiv, Ukraine.

48 Partly supported by the Russian Foundation for Basic Research, grant 11-02-91345-DFG_a. 


\section{Introduction}

The study of jet production in ep collisions at HERA has been well established as a testing ground of perturbative QCD (pQCD). Jet cross sections provided precise determinations of the strong coupling constant, $\alpha_{s}$, and its scale dependence. The jet observables used to test pQCD included inclusive-jet [1-7], dijet [1,4,6-9] and multijet [6,7,10-12] cross sections in neutral current (NC) deep inelastic ep scattering (DIS), inclusive-jet [13,14], dijet [15-20] and multijet $[21,22]$ cross sections in photoproduction and the internal structure of jets in NC [23-25] and charged current [26,27] DIS. These studies also demonstrated that the $k_{T}$ cluster algorithm [28] in the longitudinally invariant inclusive mode [29] results in the smallest uncertainties in the reconstruction of jets in ep collisions. Jet cross sections in NC DIS [2] and photoproduction [16] were used by ZEUS [30] as input in a QCD analysis to extract the parton distribution functions (PDFs) of the proton; these data helped to constrain the gluon density at medium- to high- $x$ values, where $x$ is the fraction of the proton momentum carried by the gluon.

The $k_{T}$ algorithm is well suited for $e p$ collisions and yields infrared- and collinear-safe cross sections at any order of pQCD. However, it might not be best suited to reconstruct jets in hadron-hadron collisions, such as those at the LHC. In order to optimise the reconstruction of jet observables in such environments, new infrared- and collinear-safe jet algorithms were recently developed, namely the anti- $k_{T}$ [31], a recombination-type jet algorithm, and the "Seedless Infrared-Safe" cone (SIScone) [32] algorithms. Measurements of jet cross sections in NC DIS using these algorithms were recently published [33] and constituted the first measurements with these new jet algorithms. The results tested the performance of these jet algorithms with data in a well understood hadron-induced reaction and it was shown that pQCD calculations with up to four partons in the final state provide a good description of the differences between jet algorithms.

Measurements of inclusive-jet cross sections in photoproduction are presented in this paper. Two types of QCD processes contribute to jet production in photoproduction; at leading order they can be separated into $[34,35]$ the direct process, in which the photon interacts directly with a parton in the proton, and the resolved process, in which the photon acts as a source of partons, one of which interacts with a parton in the proton. Due to the presence of the resolved processes, the analysis of jet cross sections in photoproduction with different jet algorithms provides a test of their performance in a reaction closer to hadron-hadron interactions than NC DIS.

In this paper, single-differential inclusive-jet cross sections as functions of the jet transverse energy, $E_{T}^{\mathrm{jet}}$, and pseudorapidity, $\eta^{\mathrm{jet}}$, are presented based on the $k_{T}$, anti- $k_{T}$ and SIScone jet

\footnotetext{
49 Alexander von Humboldt Professor; also at DESY and University of Oxford.

50 STFC Advanced Fellow.

51 Nee Korcsak-Gorzo.

52 This material was based on work supported by the National Science Foundation, while working at the Foundation.

53 Also at Max Planck Institute for Physics, Munich, Germany, External Scientific Member.

54 Now at Tokyo Metropolitan University, Japan.

55 Now at Nihon Institute of Medical Science, Japan.

56 Now at Osaka University, Osaka, Japan.

57 Also at Łódź University, Poland.

58 Member of Łódź University, Poland.

59 Now at Department of Physics, Stockholm University, Stockholm, Sweden.

60 Also at Cardinal Stefan Wyszyński University, Warsaw, Poland.

61 Deceased.
} 
algorithms. The results based on the anti- $k_{T}$ and SIScone jet algorithms are compared to the measurements based on the $k_{T}$ via the ratios of cross sections. In addition, measurements of cross sections are also presented as functions of $E_{T}^{\mathrm{jet}}$ in different regions of $\eta^{\text {jet }}$, which have the potential to constrain further the gluon density at high $x$. Next-to-leading-order (NLO) QCD calculations using recent parameterisations of the proton and photon PDFs are compared to the measurements. A determination of $\alpha_{S}\left(M_{Z}\right)$ as well as of its energy-scale dependence are also presented. The analyses presented here are based on a data sample with a more than three-fold increase in statistics with respect to the previous study [13].

\section{Experimental set-up}

A detailed description of the ZEUS detector can be found elsewhere [36,37]. A brief outline of the components most relevant for this analysis is given below.

Charged particles were tracked in the central tracking detector (CTD) [38], which operated in a magnetic field of $1.43 \mathrm{~T}$ provided by a thin superconducting solenoid. The CTD consisted of 72 cylindrical drift-chamber layers, organised in nine superlayers covering the polarangle ${ }^{62}$ region $15^{\circ}<\theta<164^{\circ}$. The CTD was complemented by a silicon microvertex detector (MVD) [39], consisting of three active layers in the barrel and four disks in the forward region. For CTD-MVD tracks that pass through all nine CTD superlayers, the momentum resolution was $\sigma\left(p_{T}\right) / p_{T}=0.0029 p_{T} \oplus 0.0081 \oplus 0.0012 / p_{T}$, with $p_{T}$ in $\mathrm{GeV}$.

The high-resolution uranium-scintillator calorimeter (CAL) [40] consisted of three parts: the forward (FCAL), the barrel (BCAL) and the rear (RCAL) calorimeters. Each part was subdivided transversely into towers and longitudinally into one electromagnetic section (EMC) and either one (in RCAL) or two (in BCAL and FCAL) hadronic sections (HAC). The smallest subdivision of the calorimeter was called a cell. The CAL energy resolutions, as measured under test-beam conditions, were $\sigma(E) / E=0.18 / \sqrt{E}$ for electrons and $\sigma(E) / E=0.35 / \sqrt{E}$ for hadrons, with $E$ in $\mathrm{GeV}$.

The luminosity was measured using the Bethe-Heitler reaction $e p \rightarrow e \gamma p$ by a luminosity detector which consisted of a lead-scintillator calorimeter [41] and an independent magnetic spectrometer [42]. The fractional uncertainty on the measured luminosity was $1.8 \%$.

\section{Data selection}

The data were collected during the running period 2005-2007, when HERA operated with protons of energy $E_{p}=920 \mathrm{GeV}$ and electrons or positrons ${ }^{63}$ of energy $E_{e}=27.5 \mathrm{GeV}$, at an ep centre-of-mass energy of $\sqrt{s}=318 \mathrm{GeV}$, and correspond to an integrated luminosity of $299.9 \pm 5.4 \mathrm{pb}^{-1}$.

A three-level trigger system was used to select events online [37,43]. At the first level, events were triggered by a coincidence of a regional or transverse energy sum in the CAL and at least one track from the interaction point measured in the CTD. At the second level, a total transverse energy of at least $12 \mathrm{GeV}$, excluding the energy in the eight CAL towers immediately

\footnotetext{
62 The ZEUS coordinate system was a right-handed Cartesian system, with the $Z$ axis pointing in the proton beam direction, referred to as the "forward direction", and the $X$ axis pointing towards the centre of HERA. The coordinate origin was at the nominal interaction point.

63 In the following, the term electron will refer to both the electron and positron, unless otherwise stated.
} 
surrounding the forward beampipe, ${ }^{64}$ was required, and cuts on CAL energies and timing were used to suppress events caused by interactions between the proton beam and residual gas in the beampipe. At the third level, two different methods were applied to select the events. The first method selected events with a total transverse energy of at least $25 \mathrm{GeV}$, excluding the energy in the eight CAL towers immediately surrounding the forward beampipe. For the second method, a jet algorithm was applied to the CAL cells and jets were reconstructed using the energies and positions of these cells; events with at least one jet of $E_{T}>10 \mathrm{GeV}$ and $\eta<2.5$ were accepted. Additional requirements based on CAL energies, tracking and timing were used to suppress further the non-ep background.

Events from collisions between quasi-real photons and protons were selected offline using similar criteria to those reported in the previous ZEUS publication [13]. The selection criteria applied were:

- a reconstructed event vertex along the $Z$ axis within $35 \mathrm{~cm}$ of the nominal interaction point was required;

- cuts based on tracking information were applied to remove the contamination from beamgas interactions, cosmic-ray showers and beam-halo muons;

- charged current DIS events were rejected by requiring the total missing transverse momentum, $p_{T}^{\text {miss }}$, to be small compared to the total transverse energy, $E_{T}^{\mathrm{tot}}$, i.e. $p_{T}^{\text {miss }} / \sqrt{E_{T}^{\mathrm{tot}}}<$ $2 \sqrt{\mathrm{GeV}}$

- any NC DIS event with an identified scattered-electron candidate in the CAL was rejected;

- the events were restricted to $\gamma p$ centre-of-mass energies in the region $142<W_{\gamma p}<$ $293 \mathrm{GeV}$, where $W_{\gamma p}=\sqrt{s y} ; y$ is the inelasticity and was estimated as $y_{\mathrm{JB}}=\left(E-p_{Z}\right) / 2 E_{e}$, where $E$ is the total energy measured in the CAL and $p_{Z}$ is the longitudinal component of the total momentum.

After these selection criteria were applied, the contamination from beam-gas interactions, cosmic-ray showers and beam-halo muons was found to be negligible. The remaining background from NC DIS events was estimated by Monte Carlo (MC) techniques to be around 1\% and was neglected. The contamination from charged current DIS events was found to be even smaller. The selected sample consisted of events from ep interactions with $Q^{2}<1 \mathrm{GeV}^{2}$, where $Q^{2}$ is the virtuality of the exchanged photon, and a median $Q^{2} \approx 10^{-4} \mathrm{GeV}^{2}$, estimated using MC techniques.

\section{Jet search}

In photoproduction, jets are usually defined using the transverse-energy flow in the pseudorapidity-azimuth $(\eta-\phi)$ plane of the laboratory frame $[28,29,44]$. The procedure to reconstruct jets with the $k_{T}$ algorithm from an initial list of objects (e.g. final-state partons, final-state hadrons or energy deposits in the calorimeter) is described below in some detail. In the following discussion, $E_{T}^{i}$ denotes the transverse energy, $\eta^{i}$ the pseudorapidity and $\phi^{i}$ the azimuthal angle of object $i$. For each pair of objects, the quantity

$$
d_{i j}=\min \left(\left(E_{T}^{i}\right)^{2},\left(E_{T}^{j}\right)^{2}\right) \cdot\left[\left(\eta^{i}-\eta^{j}\right)^{2}+\left(\phi^{i}-\phi^{j}\right)^{2}\right] / R^{2}
$$

\footnotetext{
64 The corresponding range in pseudorapidity is $2.7 \lesssim \eta \lesssim 3.8$.
} 
is calculated, where $R$, referred to as "jet radius", represents the maximum distance in the $\eta-\phi$ plane for particle recombination. For each individual object, the distance to the beam, $d_{i}=\left(E_{T}^{i}\right)^{2}$, is also calculated. If, of all the values $\left\{d_{i j}, d_{i}\right\}, d_{k l}$ is the smallest, then objects $k$ and $l$ are combined into a single new object. If, however, $d_{k}$ is the smallest, then object $k$ is considered a jet and removed from the sample. The procedure is repeated until all objects are assigned to jets.

The anti- $k_{T}$ algorithm is identical to the $k_{T}$ except for a modified distance measure,

$$
d_{i j}=\min \left(\left(E_{T}^{i}\right)^{-2},\left(E_{T}^{j}\right)^{-2}\right) \cdot\left[\left(\eta^{i}-\eta^{j}\right)^{2}+\left(\phi^{i}-\phi^{j}\right)^{2}\right] / R^{2},
$$

and the distance to the beam, which is defined as $d_{i}=\left(E_{T}^{i}\right)^{-2}$.

The SIScone algorithm consists of two steps. First, for a given set of initial objects, all stable cones are identified; cones are classified as stable by the coincidence of the cone axis with that defined by the total momentum of the objects contained in the given cone of radius $R$ in the $\eta-\phi$ plane. In this procedure, no seed is used. Stable cones are then discarded if their transverse momentum is below a given threshold, $p_{t, \min }$. For each selected stable cone, the scalar sum of the transverse momentum of the objects associated to it, $\tilde{p}_{t}$, is defined. Second, overlapping cones are identified and subsequently split or merged according to the following procedure. Two cones are merged if the scalar sum of the transverse momentum of the objects shared by the two cones exceeds a certain fraction $f$ of the lowest- $\tilde{p}_{t}$ cone; otherwise, two different cones are considered and the common objects are assigned to the nearest cone.

For the measurements presented in this paper, the jet radius $R$ was set to unity and the jet variables were defined according to the Snowmass convention [45] for all three jet algorithms. In the application of the SIScone algorithm, the fraction $f$ was set to 0.75 and $p_{t}$, min was set to zero.

The $k_{T}$, anti- $k_{T}$ and SIScone jet algorithms ${ }^{65}$ were used to reconstruct jets in the hadronic final state from the energy deposits in the CAL cells. The jets reconstructed from the CAL cell energies are called calorimetric jets and the variables associated with them are denoted by $E_{T \text {,cal }}^{\text {jet }}, \eta_{\text {cal }}^{\text {jet }}$ and $\phi_{\mathrm{cal}}^{\text {jet }}$. Three samples of events were selected for further analysis, one for each jet algorithm, which contain at least one jet satisfying $E_{T, \text { cal }}^{\text {jet }}>13 \mathrm{GeV}$ and $-1<\eta_{\text {cal }}^{\text {jet }}<2.5$.

\section{Monte Carlo simulations}

Samples of events were generated to determine the response of the detector to jets of hadrons and the correction factors necessary to obtain the hadron-level jet cross sections. In addition, these samples were used to estimate hadronisation corrections to the NLO calculations (see Section 8).

The MC programs Pythia 6.146 [47] and HeRwig 6.504 [48] were used to generate resolved and direct photoproduction events. In both generators, the partonic processes are simulated using leading-order matrix elements, with the inclusion of initial- and final-state parton showers. Fragmentation into hadrons was performed using the Lund string model [49], as implemented in JetSET [50] in the case of Pythia, and a cluster model [51] in the case of HeRWIG. The CTEQ4M [52] (GRV-HO [53]) sets were used for the proton (photon) PDFs. Samples of PYTHIA including multiparton interactions [54] (PYTHIA-MI) with a minimum transverse momentum

$\overline{65}$ The FAstJet 2.4.1 [46] package was used. 
Table 1

Number of events and jets selected in data with $E_{T}^{\text {jet }}>17 \mathrm{GeV}$ and $-1<\eta^{\text {jet }}<2.5$ in the kinematic region of $Q^{2}<1 \mathrm{GeV}^{2}$ and $142<W_{\gamma p}<293 \mathrm{GeV}$ for the $k_{T}$, anti- $k_{T}$ and SIScone jet algorithms. The number of events with one, two, three and four jets are also listed.

\begin{tabular}{lrrr}
\hline & \multicolumn{1}{c}{$k_{T}$} & anti- $k_{T}$ & SIScone \\
\hline Events & 483328 & 444295 & 438906 \\
Jets & 613165 & 572865 & 566000 \\
One jet & 355691 & 317509 & 313519 \\
Two jets & 125468 & 125016 & 123700 \\
Three jets & 2138 & 1756 & 1667 \\
Four jets & 31 & 14 & 20 \\
\hline
\end{tabular}

for the secondary scatter, $p_{T \text {, min }}^{\mathrm{sec}}$, of $1,1.5$ or $2 \mathrm{GeV}$ were used to simulate contributions from non-perturbative effects not related to hadronisation (NP), such as the underlying event. All the samples of generated events were passed through the GEANT 3.21-based [55] ZEUS detectorand trigger-simulation programs [37]. They were reconstructed and analysed by the same program chain as the data.

The jet search was performed on the MC events using the energy measured in the CAL cells as described in Section 4. In addition, the three jet algorithms were also applied to the final-state particles (hadron level) and partons (parton level). The hadron level is defined by those hadrons with lifetime $\tau \geqslant 10$ ps and the parton level is defined as those partons present after the partonshower procedure.

\section{Transverse-energy and acceptance corrections}

The comparison of the reconstructed jet variables for the hadronic and the calorimetric jets in MC-simulated events showed that no correction was needed for the jet pseudorapidity and azimuth. However, $E_{T, \text { cal }}^{\text {jet }}$ underestimates the corresponding hadronic-jet transverse energy by $\approx 14 \%$ with an r.m.s. of $\approx 10 \%$. This underestimation is mainly due to the energy lost by the particles in the inactive material in front of the CAL. The transverse-energy corrections to calorimetric jets, as functions of $\eta_{\text {cal }}^{\text {jet }}$ and $E_{T \text {,cal }}^{\text {jet }}$ and averaged over $\phi_{\text {cal }}^{\text {jet }}$, were determined using the $\mathrm{MC}$ events. Further corrections to the jet transverse energy were applied to the data to account for differences in the jet energy scale between data and MC simulations; the method presented previously $[17,56]$, which relies on a good understanding of the performance of the track reconstruction, was used to calibrate the absolute energy scale of the jets down to $\pm 1 \%$. This calibration was cross checked by means of the transverse-momentum balance in the CAL between the electron candidate and the jet in single-jet NC DIS events.

Henceforth, jet variables without subscript refer to the corrected values. After all these corrections to the jet transverse energy, events with at least one jet satisfying $E_{T}^{\mathrm{jet}}>17 \mathrm{GeV}$ and $-1<\eta^{\text {jet }}<2.5$ were retained. The number of events and jets in the final data samples are shown in Table 1 for each jet algorithm. No events with more than four jets were found in these samples.

The $E_{T}^{\text {jet }}$ and $\eta^{\text {jet }}$ distributions in the data were corrected for detector effects using bin-by-bin acceptance correction factors determined using the MC samples. These correction factors take into account the efficiency of the trigger, the selection criteria and the purity and efficiency of the jet reconstruction. For this approach to be valid, the uncorrected distributions of the data must be adequately described by the MC simulations at the detector level. This condition was satisfied by both the PYTHIA and HERWIG MC samples. The average between the acceptance correction 
factors obtained from PYTHIA and HERWIG was used to correct the data to the hadron level. The deviations in the results obtained by using either PYTHIA or HERWIG to correct the data from their average were taken to represent systematic uncertainties of the effect of the QCD-cascade and hadronisation models in the corrections (see Section 7). The acceptance correction factors differed from unity by typically less than $20 \%$.

\section{Experimental uncertainties}

The following sources of systematic uncertainty were considered for the measured cross sections:

- the differences in the results obtained by using either PYTHIA or HERWIG to correct the data for detector effects. The resulting uncertainty was typically below $\pm 4 \%$;

- the effect of the CAL energy-scale uncertainty on $W_{\gamma p}$ was estimated by varying $y_{\mathrm{JB}}$ by $\pm 1 \%$ in simulated events. The uncertainty in the cross sections was below $\pm 1 \%$ at low $E_{T}^{\mathrm{jet}}$, increasing to $\approx \pm 3 \%$ at high $E_{T}^{\mathrm{jet}}$;

- the effect of the uncertainty on the parameterisations of the proton and photon PDFs was estimated by using alternative sets of PDFs (MRST(c-g) [57] for the proton and AFG-HO [58] for the photon) in the MC simulation to compute the acceptance correction factors. The variation of the cross sections was typically smaller than $\pm 1 \%$;

- the uncertainty in the cross sections due to that in the simulation of the trigger was found to be negligible.

All the above systematic uncertainties were added in quadrature and are shown in the figures as error bars. The resulting systematic uncertainty in the cross sections based on the three jet algorithms was similar and typically below $\pm 5 \%$.

The absolute energy scale of the calorimetric jets in simulated events was varied by its uncertainty of $\pm 1 \%$ (see Section 6); the effect of this variation on the inclusive-jet cross sections was typically $\mp 5 \%$ at low $E_{T}^{\mathrm{jet}}$, increasing up to $\mp 10 \%$ at high $E_{T}^{\mathrm{jet}}$. This uncertainty is fully correlated between measurements in different bins and is shown separately as a shaded band in the figures. In addition, there was an overall normalisation uncertainty of $\pm 1.8 \%$ from the luminosity determination, which is not included in the figures and tables.

\section{Next-to-leading-order QCD calculations}

The NLO QCD $\left(\mathcal{O}\left(\alpha_{s}^{2}\right)\right)$ calculations used in the analysis presented here were computed using the program by Klasen, Kleinwort and Kramer [59]. The calculations use the phase-space-slicing method [60] with an invariant-mass cut to isolate the singular regions of the phase space. The number of flavours was set to five and the renormalisation $\left(\mu_{R}\right)$ and factorisation $\left(\mu_{F}\right)$ scales were chosen to be $\mu_{R}=\mu_{F}=\mu=E_{T}^{\text {jet }}$. The strong coupling constant was calculated at two loops with $\Lambda_{\overline{\mathrm{MS}}}=226 \mathrm{MeV}$, corresponding to $\alpha_{S}\left(M_{Z}\right)=0.118$. The calculations were performed using the ZEUS-S [61] parameterisations of the proton PDFs and the GRV-HO sets for the photon PDFs as default. ${ }^{66}$ The three jet algorithms were applied to the partons in the events

$\overline{66}$ The LHAPDF 5.7.1 [62] package was used. 
generated by this program to compute the jet cross-section predictions. At $\mathcal{O}\left(\alpha_{s}^{2}\right)$, the partonlevel predictions from the $k_{T}$ and anti- $k_{T}$ jet algorithms are identical. The predictions from the $k_{T}$ and SIScone jet algorithms start to differ at this order.

Since the measurements refer to jets of hadrons, whereas the NLO QCD calculations refer to jets of partons, the predictions were corrected to the hadron level using the MC models. The multiplicative correction factor, $C_{\text {had }}$, was defined as the ratio of the cross section for jets of hadrons over that for jets of partons, estimated by using the MC programs described in Section 5. The mean of the ratios obtained with PyThia and HeRwig was taken as the value of $C_{\text {had }}$. Details on the values of $C_{\text {had }}$ are presented in Section 9.

The following sources of uncertainty in the theoretical predictions were considered:

- the uncertainty on the NLO QCD calculations due to that on the value of $\alpha_{S}\left(M_{Z}\right)$ used was estimated by repeating the calculations using two additional ZEUS-S sets of proton PDFs, for which different values of $\alpha_{S}\left(M_{Z}\right)$ were assumed in the fits. The difference between these calculations was used to determine the uncertainty due to that on the value of $\alpha_{s}\left(M_{Z}\right)[63]$;

- the uncertainty on the NLO QCD calculations due to terms beyond NLO was estimated by repeating the calculations using values of $\mu$ scaled by factors 0.5 and 2;

- the uncertainty from the modelling of the QCD-cascade and hadronisation effects was assumed to be half the difference between the hadronisation corrections obtained using the PYTHIA and HeRWIG models;

- the uncertainty on the NLO QCD calculations due to those on the proton PDFs was estimated by repeating the calculations using 22 additional sets from the ZEUS-S error analysis with $\alpha_{S}\left(M_{Z}\right)$ fixed to the central value; this error analysis takes into account the statistical and correlated experimental uncertainties of each data set used in the determination of the proton PDFs;

- the uncertainty on the NLO QCD calculations due to those on the photon PDFs was estimated by using alternative sets of parameterisations, AFG04 [64] and CJK [65].

The total theoretical uncertainty was obtained by adding in quadrature the individual uncertainties listed above. Fig. 1 shows an overview of the relative theoretical uncertainties for the inclusive-jet cross sections in the kinematic region of the measurements as functions of $\eta^{\text {jet }}$ and $E_{T}^{\mathrm{jet}}$ and for each jet algorithm separately. The uncertainty due to higher orders is somewhat larger for the SIScone than for the $k_{T}$ and anti- $k_{T}$ algorithms, whereas the other uncertainties are very similar for the three jet algorithms. The uncertainty coming from the terms beyond NLO is dominant in all cases. At high $E_{T}^{\text {jet }}$, the proton PDF uncertainty is of the same order (slightly smaller) as that arising from terms beyond NLO for the $k_{T}$ and anti- $k_{T}$ (SIScone) algorithms. The uncertainty arising from the photon PDFs at high $\eta^{\text {jet }}$ becomes comparable to that coming from higher orders. The uncertainties from the value of $\alpha_{S}\left(M_{Z}\right)$ and hadronisation corrections are small.

The samples of PyTHIA-MI described in Section 5 were used to estimate the contribution from non-perturbative effects not related to hadronisation. Such effects were computed as ratios of the cross section for jets of hadrons in the PYTHIA-MI samples over that for the samples of PYTHIA; these ratios are called $C_{\mathrm{NP}}$. The values of $C_{\mathrm{NP}}$ depend strongly on $p_{T, \min }^{\mathrm{sec}}$, the minimum

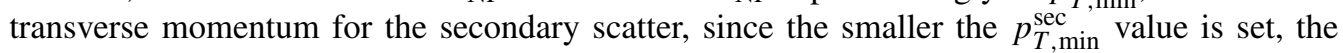
larger the phase space available for production of secondary interactions and hence the higher the jet rate. Another feature of such secondary interactions is that, due to the particular kinematics 


\section{ZEUS}
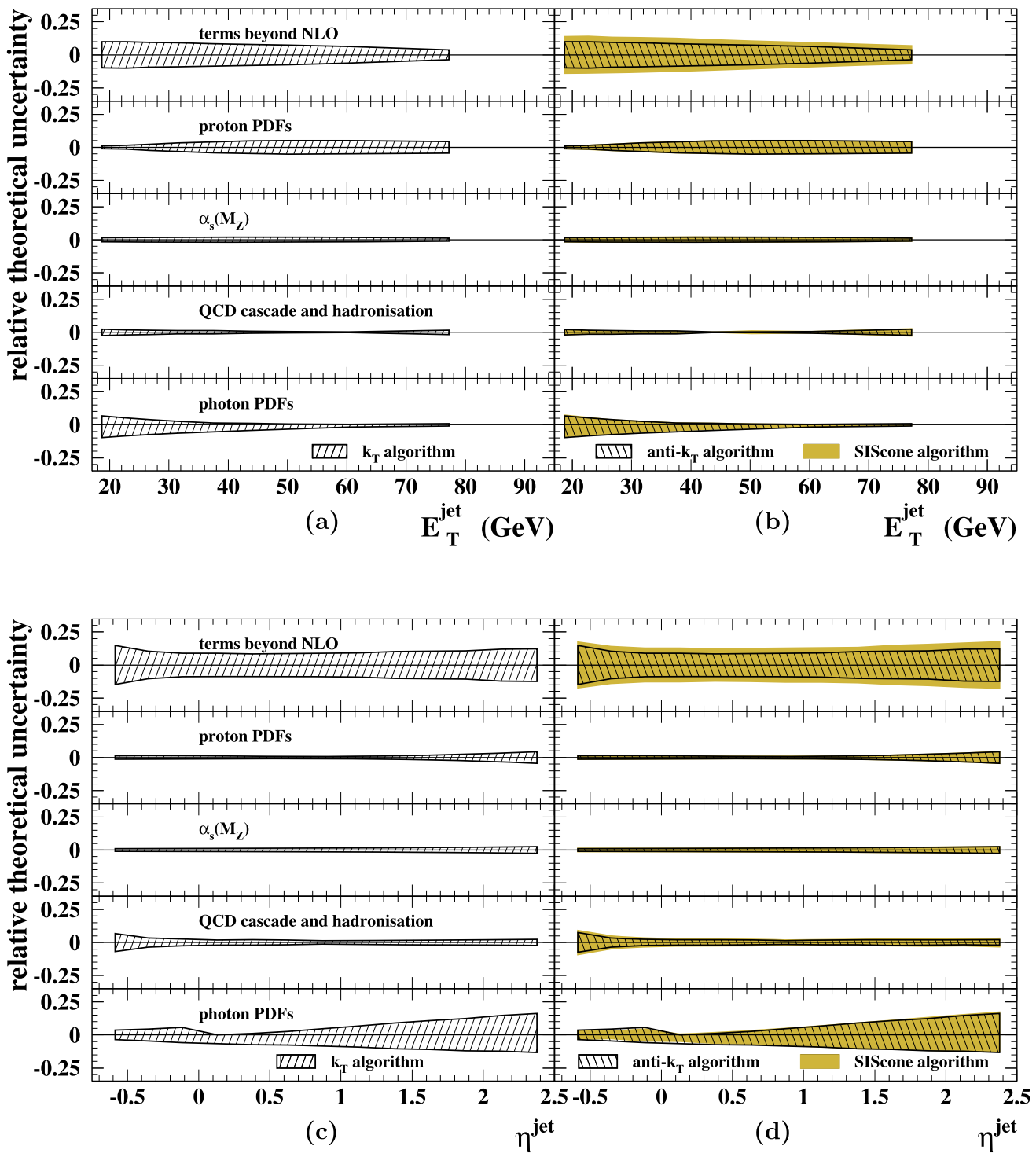

Fig. 1. Overview of the relative theoretical uncertainties for inclusive-jet cross sections in photoproduction in the kinematic region of the measurements as functions of (a,b) $E_{T}^{\text {jet }}$ and (c, d) $\eta \eta^{\text {jet }}$ for the $k_{T}$, anti- $k_{T}$ and SIScone jet algorithms. Shown are the relative uncertainties induced by the terms beyond NLO, the proton PDFs, the value of $\alpha_{S}\left(M_{Z}\right)$, the modelling of the QCD-cascade and hadronisation and the photon PDFs.

of HERA, the products of these additional interactions are expected to be boosted towards the proton direction. Several predictions including these non-perturbative effects, denoted as NLO $\otimes$ $\mathrm{NP}$, were computed by applying the factors $C_{\mathrm{NP}}$, using the $p_{T, \min }^{\mathrm{sec}}=1,1.5$ and $2 \mathrm{GeV}$ PYTHIAMI samples, to the NLO QCD calculations after hadronisation corrections. 


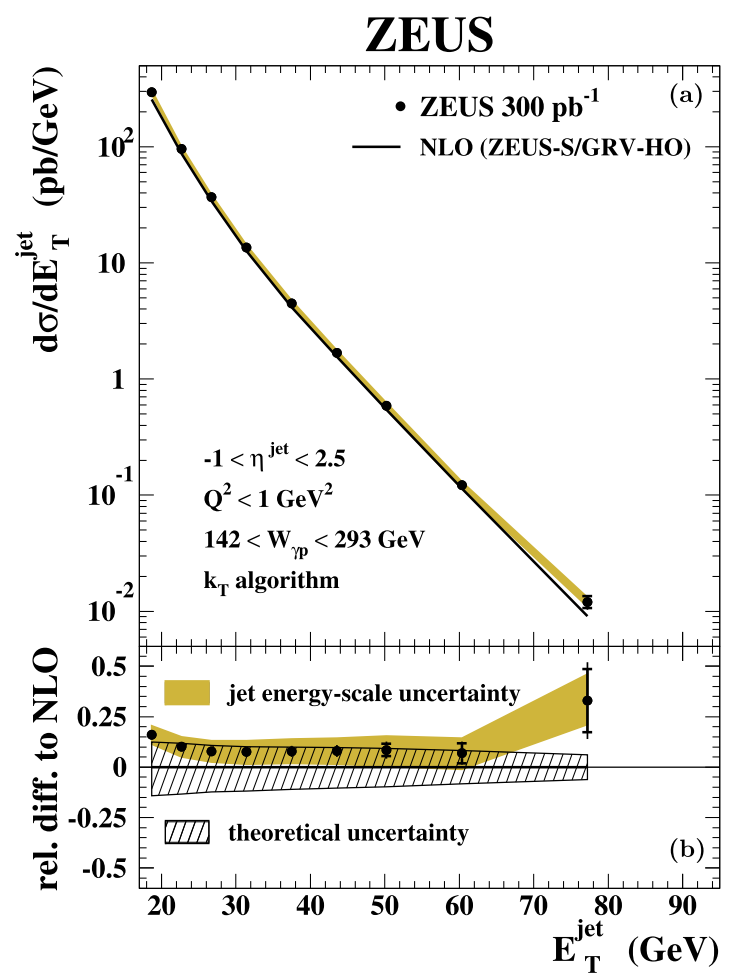

Fig. 2. (a) The measured differential cross section $d \sigma / d E_{T}^{\text {jet }}$ based on the $k_{T}$ jet algorithm for inclusive-jet photoproduction with $-1<\eta^{\text {jet }}<2.5$ (dots) in the kinematic region given by $Q^{2}<1 \mathrm{GeV}^{2}$ and $142<W_{\gamma p}<293 \mathrm{GeV}$. The NLO QCD calculation (solid line), corrected to include hadronisation effects and using the ZEUS-S (GRV-HO) parameterisations of the proton (photon) PDFs, is also shown. (b) The relative difference between the measured $d \sigma / d E_{T}^{\mathrm{jet}}$ and the NLO QCD calculation (dots). In both figures, the inner error bars represent the statistical uncertainties; the outer error bars show the statistical and systematic uncertainties not associated with the uncertainty in the absolute energy scale of the jets, added in quadrature; the shaded band displays the uncertainty due to the absolute energy scale of the jets and the hatched band displays the total theoretical uncertainty. In some bins, the error bars on the data points are smaller than the marker size and are therefore not visible.

\section{Results}

Single- and double-differential inclusive-jet cross sections were measured in the kinematic region given by $Q^{2}<1 \mathrm{GeV}^{2}$ and $142<W_{\gamma p}<293 \mathrm{GeV}$. These cross sections include every jet of hadrons with $E_{T}^{\text {jet }}>17 \mathrm{GeV}$ and $-1<\eta^{\text {jet }}<2.5$ in each event. The jets were reconstructed using either the $k_{T}$, the anti- $k_{T}$ or the SIScone jet algorithms. The $x$ region covered by the measurements was determined to be $3 \cdot 10^{-3}<x<0.95$.

\subsection{Single-differential cross sections}

The measurements of the single-differential cross sections based on the $k_{T}$ jet algorithm as functions of $E_{T}^{\mathrm{jet}}$ and $\eta^{\mathrm{jet}}$ are presented in Figs. 2 and 3 and Table 2. In these and the subsequent figures, each data point is plotted at the weighted mean of each bin. The measured $d \sigma / d E_{T}^{\text {jet }}$ 


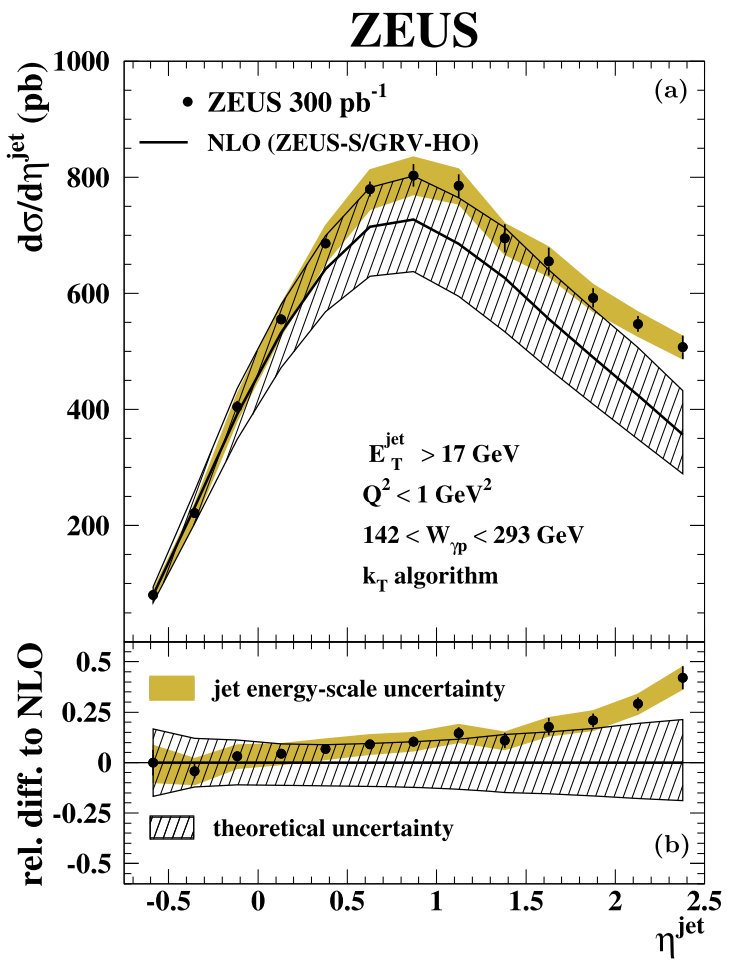

Fig. 3. (a) The measured differential cross section $d \sigma / d \eta^{\text {jet }}$ based on the $k_{T}$ jet algorithm for inclusive-jet photoproduction with $E_{T}^{\text {jet }}>17 \mathrm{GeV}$ (dots) in the kinematic region given by $Q^{2}<1 \mathrm{GeV}^{2}$ and $142<W_{\gamma p}<293 \mathrm{GeV}$. (b) The relative difference between the measured $d \sigma / d \eta^{\text {jet }}$ and the NLO QCD calculation (dots). Other details as in the caption to Fig. 2.

falls by over four orders of magnitude in the measured range. The measured $d \sigma / d \eta^{\text {jet }}$ displays a maximum around $\eta^{\text {jet }} \approx 1$.

The NLO QCD predictions are compared to the measurements in these figures. The calculation reproduces the measured $d \sigma / d E_{T}^{\mathrm{jet}}$ well. The measured $d \sigma / d \eta^{\text {jet }}$ is well described for $\eta^{\text {jet }} \lesssim 2$; however, an excess of data with respect to the theory is observed for larger $\eta^{\text {jet }}$ values. Such discrepancies have already been observed in previous studies of jet photoproduction [20, 22]. In the study of dijet production [20], the discrepancies were interpreted as an inadequacy of the parameterisations of the photon PDFs, which had been extracted from $e^{+} e^{-}$data at lower scales. The studies of multijet production [22] showed the need to include non-perturbative effects not related to hadronisation in the $\mathrm{pQCD}$ calculations to describe the data.

The influence of non-perturbative effects not related to hadronisation in the predictions was investigated by using the NLO $\otimes$ NP QCD calculations (see Section 8). The comparison of these calculations to the data is shown in Fig. 4. It is observed that the NLO $\otimes$ NP QCD calculations predict a larger jet rate at low $E_{T}^{\mathrm{jet}}$ and high $\eta^{\text {jet }}$, in the region where the NLO QCD prediction fails to describe the data. The NLO $\otimes$ NP QCD prediction with $p_{T, \text { min }}^{\mathrm{sec}}=1.5 \mathrm{GeV}$ is closest to the data. These observations indicate the possible presence of effects such as the underlying event in the data, which are not included in the NLO QCD calculation. These non-perturbative contributions are expected to be unrelated to the hard scattering and approximately constant 
Table 2

The measured differential cross sections $d \sigma / d E_{T}^{\text {jet }}$ and $d \sigma / d \eta^{\text {jet }}$ based on the $k_{T}$ jet algorithm for inclusive-jet photoproduction with $-1<\eta^{\text {jet }}<2.5$ and $E_{T}^{\text {jet }}>17 \mathrm{GeV}$ in the kinematic region given by $Q^{2}<1 \mathrm{GeV}^{2}$ and $142<W_{\gamma p}<$ $293 \mathrm{GeV}$. The statistical $\left(\delta_{\text {stat }}\right)$, uncorrelated systematic $\left(\delta_{\text {syst }}\right)$ and jet-energy scale $\left(\delta_{\mathrm{ES}}\right)$ uncertainties are shown separately. The corrections for hadronisation effects to be applied to the parton-level NLO QCD calculations $\left(C_{\text {had }}\right)$ are shown in the last column.

\begin{tabular}{|c|c|c|c|c|c|c|}
\hline$E_{T}^{\mathrm{jet}} \operatorname{bin}(\mathrm{GeV})$ & $\left\langle E_{T}^{\mathrm{jet}}\right\rangle(\mathrm{GeV})$ & $d \sigma / d E_{T}^{\mathrm{jet}}(\mathrm{pb} / \mathrm{GeV})$ & $\delta_{\text {stat }}$ & $\delta_{\text {syst }}$ & $\delta_{\mathrm{ES}}$ & $C_{\text {had }}$ \\
\hline $17-21$ & 18.7 & 295.84 & \pm 0.52 & $\begin{array}{l}+6.37 \\
-6.36\end{array}$ & $\begin{array}{l}+11.35 \\
-11.93\end{array}$ & 0.99 \\
\hline $21-25$ & 22.7 & 95.86 & \pm 0.28 & $\begin{array}{l}+1.56 \\
-1.55\end{array}$ & $\begin{array}{l}+4.19 \\
-4.50\end{array}$ & 0.99 \\
\hline $25-29$ & 26.7 & 36.88 & \pm 0.18 & $\begin{array}{l}+0.52 \\
-0.52\end{array}$ & $\begin{array}{l}+1.76 \\
-1.80\end{array}$ & 0.98 \\
\hline $29-35$ & 31.4 & 13.606 & \pm 0.090 & $\begin{array}{l}+0.150 \\
-0.142\end{array}$ & $\begin{array}{l}+0.687 \\
-0.772\end{array}$ & 0.98 \\
\hline $35-41$ & 37.5 & 4.492 & \pm 0.051 & $\begin{array}{l}+0.104 \\
-0.102\end{array}$ & $\begin{array}{l}+0.250 \\
-0.251\end{array}$ & 0.98 \\
\hline $41-47$ & 43.6 & 1.677 & \pm 0.032 & $\begin{array}{l}+0.033 \\
-0.033\end{array}$ & $\begin{array}{l}+0.098 \\
-0.102\end{array}$ & 0.98 \\
\hline $47-55$ & 50.2 & 0.589 & \pm 0.017 & $\begin{array}{l}+0.015 \\
-0.015\end{array}$ & $\begin{array}{l}+0.038 \\
-0.044\end{array}$ & 0.98 \\
\hline $55-71$ & 60.3 & 0.1216 & \pm 0.0057 & $\begin{array}{l}+0.0033 \\
-0.0028\end{array}$ & $\begin{array}{l}+0.0086 \\
-0.0090\end{array}$ & 0.97 \\
\hline $71-95$ & 77.2 & 0.0121 & \pm 0.0014 & $\begin{array}{l}+0.0010 \\
-0.0010\end{array}$ & $\begin{array}{l}+0.0012 \\
-0.0011\end{array}$ & 1.00 \\
\hline$\eta^{\text {jet }}$ bin & $\left\langle\eta^{\text {jet }}\right\rangle$ & $d \sigma / d \eta^{\text {jet }}(\mathrm{pb})$ & $\delta_{\text {stat }}$ & $\delta_{\text {syst }}$ & $\delta_{\mathrm{ES}}$ & $C_{\text {had }}$ \\
\hline-0.75 to -0.50 & -0.59 & 80.65 & \pm 0.92 & $\begin{array}{l}+5.12 \\
-4.89\end{array}$ & $\begin{array}{l}+6.86 \\
-7.78\end{array}$ & 0.82 \\
\hline-0.50 to -0.25 & -0.36 & 221.4 & \pm 1.6 & $\begin{array}{l}+8.7 \\
-8.5\end{array}$ & $\begin{array}{l}+14.0 \\
-15.3\end{array}$ & 0.93 \\
\hline-0.25 to +0.00 & -0.11 & 404.4 & \pm 2.2 & $\begin{array}{l}+10.0 \\
-9.9\end{array}$ & $\begin{array}{l}+21.7 \\
-23.1\end{array}$ & 0.97 \\
\hline+0.00 to +0.25 & +0.13 & 555.2 & \pm 2.6 & $\begin{array}{l}+8.1 \\
-7.9\end{array}$ & $\begin{array}{l}+25.9 \\
-28.2\end{array}$ & 0.98 \\
\hline+0.25 to +0.50 & +0.38 & 685.8 & \pm 2.9 & $\begin{array}{l}+8.2 \\
-8.0\end{array}$ & $\begin{array}{l}+30.3 \\
-32.5\end{array}$ & 0.98 \\
\hline+0.50 to +0.75 & +0.63 & 779.6 & \pm 3.1 & $\begin{array}{l}+12.3 \\
-12.2\end{array}$ & $\begin{array}{l}+32.5 \\
-34.4\end{array}$ & 0.98 \\
\hline+0.75 to +1.00 & +0.87 & 803.0 & \pm 3.2 & $\begin{array}{l}+19.3 \\
-19.3\end{array}$ & $\begin{array}{l}+31.4 \\
-32.8\end{array}$ & 0.99 \\
\hline+1.00 to +1.25 & +1.12 & 784.9 & \pm 3.3 & $\begin{array}{l}+19.6 \\
-19.6\end{array}$ & $\begin{array}{l}+29.1 \\
-30.7\end{array}$ & 0.99 \\
\hline+1.25 to +1.50 & +1.38 & 694.9 & \pm 2.9 & $\begin{array}{l}+24.0 \\
-24.0\end{array}$ & $\begin{array}{l}+25.1 \\
-27.0\end{array}$ & 1.00 \\
\hline+1.50 to +1.75 & +1.63 & 654.8 & \pm 2.8 & $\begin{array}{l}+23.8 \\
-23.8\end{array}$ & $\begin{array}{l}+24.6 \\
-25.3\end{array}$ & 1.00 \\
\hline+1.75 to +2.00 & +1.88 & 592.1 & \pm 2.6 & $\begin{array}{l}+16.4 \\
-16.3\end{array}$ & $\begin{array}{l}+22.5 \\
-23.2\end{array}$ & 1.00 \\
\hline+2.00 to +2.25 & +2.13 & 547.3 & \pm 2.6 & $\begin{array}{l}+13.4 \\
-13.4\end{array}$ & $\begin{array}{l}+20.4 \\
-21.1\end{array}$ & 1.00 \\
\hline+2.25 to +2.50 & +2.38 & 507.2 & \pm 2.4 & $\begin{array}{l}+20.2 \\
-20.2 \\
\end{array}$ & $\begin{array}{l}+18.7 \\
-19.9 \\
\end{array}$ & 1.00 \\
\hline
\end{tabular}

with the scale of the interaction, so that the ratio of this non-perturbative contribution to the jet transverse energy becomes smaller as $E_{T}^{\mathrm{jet}}$ increases, as seen in Fig. 4a. This is supported by the good description of the data by the NLO QCD calculation for $E_{T}^{\mathrm{jet}}>21 \mathrm{GeV}$ (Fig. 4a) and by the inclusive-jet cross section as a function of $\eta^{\text {jet }}$ for $E_{T}^{\text {jet }}>21 \mathrm{GeV}$ (see Fig. 5 and Table 3): the NLO QCD calculation gives a good description of the data in the whole measured range; in particular, discrepancies between data and theory are no longer observed at high values of $\eta^{\text {jet }}$. In addition, the differences between the $\mathrm{NLO} \otimes \mathrm{NP}$ predictions with different $p_{T, \text { min }}^{\mathrm{sec}}$ values become smaller, as seen in Fig. 5b. 


\section{ZEUS}
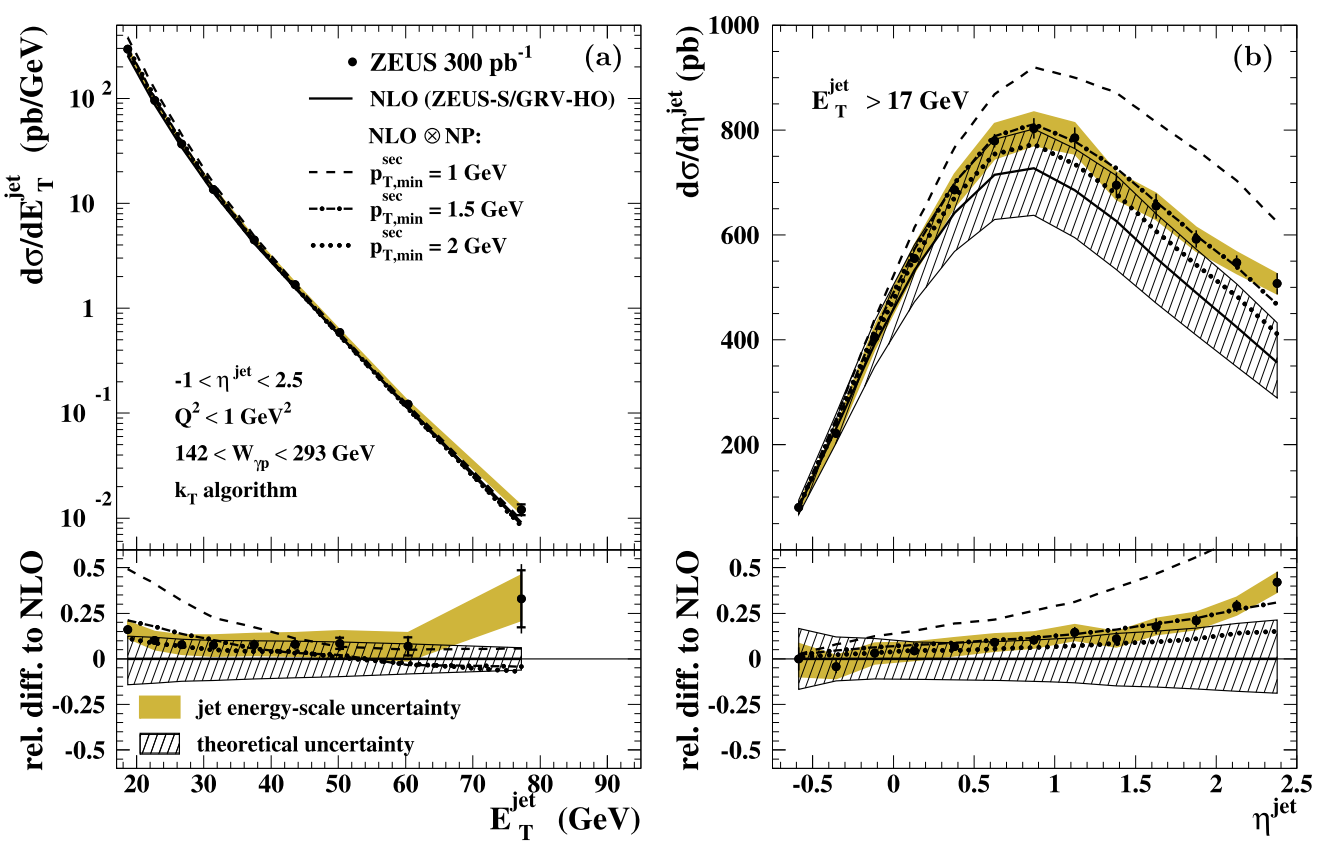

Fig. 4. The measured differential cross sections (a) $d \sigma / d E_{T}^{\text {jet }}$ and (b) $d \sigma / d \eta^{\text {jet }}$ based on the $k_{T}$ jet algorithm for inclusive-jet photoproduction with $E_{T}^{\text {jet }}>17 \mathrm{GeV}$ and $-1<\eta^{\text {jet }}<2.5$ (dots) in the kinematic region given by $Q^{2}<1 \mathrm{GeV}^{2}$ and $142<W_{\gamma p}<293 \mathrm{GeV}$. For comparison, the NLO QCD calculations including an estimation of non-perturbative effects (see text) are also shown. Other details as in the caption to Fig. 2.

The influence of the poorly constrained photon PDFs on the predictions was investigated by comparing calculations based on different PDF sets to the data. Fig. 6 shows the measurements together with the NLO QCD predictions using alternatively the AFG04 and CJK sets of photon PDFs, together with the predictions based on the GRV-HO set. Some differences are observed between the three predictions, especially at low $E_{T}^{\text {jet }}$ and high $\eta^{\text {jet }}$. In particular, the predictions based on AFG04 (CJK) are lower (higher) than those based on GRV-HO.

The influence of the proton PDFs on the predictions was investigated by comparing calculations based on different PDF sets to the data. Fig. 7 shows the measurements together with the NLO QCD predictions using alternatively the MSTW08 [66] and HERAPDF1.5 [67] sets of proton PDFs, together with the predictions based on the ZEUS-S set. The prediction based on HERAPDF1.5 is lower than that based on the ZEUS-S set in most of the phase space, whereas the MSTW08 prediction is higher at high $E_{T}^{\mathrm{jet}}$. This region of phase space is not well constrained since the main contribution comes from the high- $x$ gluon density in the proton.

In summary, the measurements of inclusive-jet cross sections in photoproduction have the potential to constrain the proton and the photon PDFs. To study in more detail the sensitivity of the inclusive-jet cross sections to the proton and photon PDFs and find the regions of phase space in which the data can add information to constrain further these PDFs, double-differential cross sections were measured and are presented in the next section. 


\section{RAPID COMMUNICATION}

\section{ZEUS}
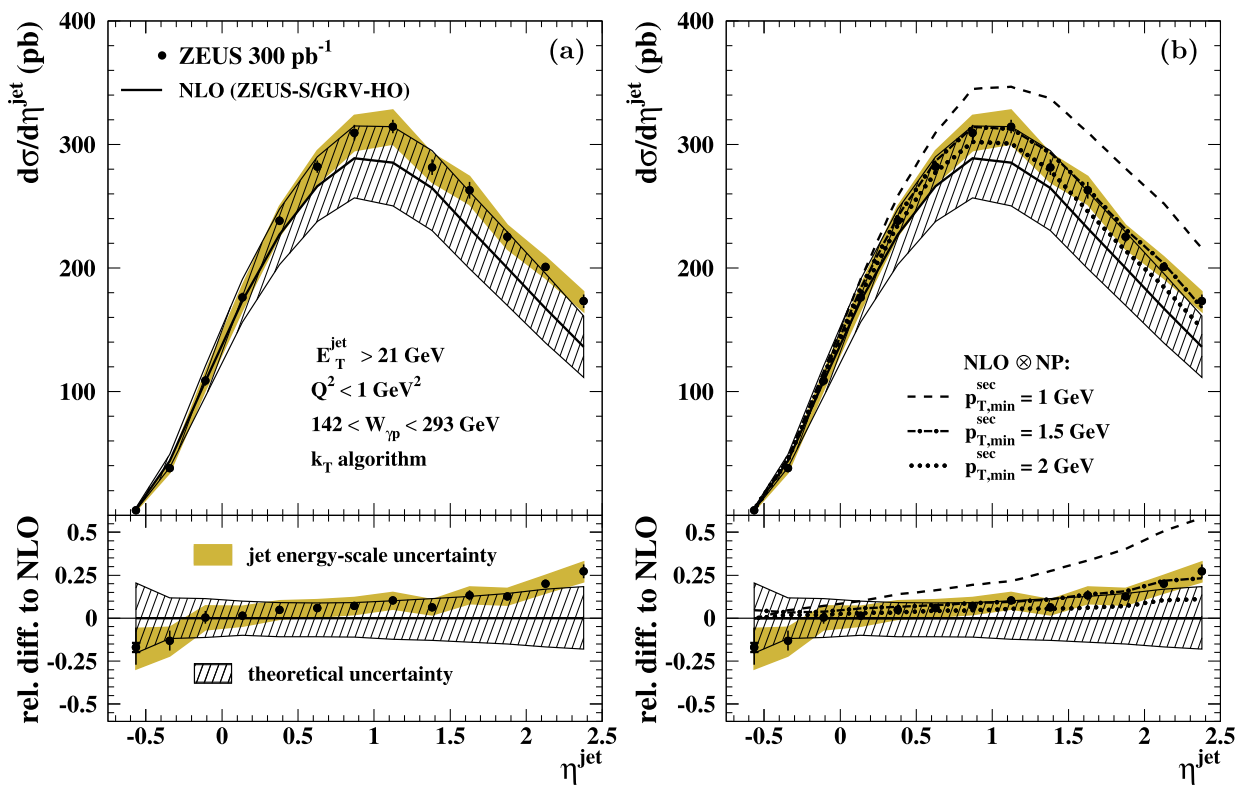

Fig. 5. The measured differential cross section $d \sigma / d \eta^{\text {jet }}$ based on the $k_{T}$ jet algorithm for inclusive-jet photoproduction with $E_{T}^{\text {jet }}>21 \mathrm{GeV}$ (dots) in the kinematic region given by $Q^{2}<1 \mathrm{GeV}^{2}$ and $142<W_{\gamma p}<293 \mathrm{GeV}$. In (b), the NLO QCD calculations including an estimation of non-perturbative effects (see text) are also shown. Other details as in the caption to Fig. 2.

Table 3

The measured differential cross sections $d \sigma / d \eta^{\text {jet }}$ based on the $k_{T}$ jet algorithm for inclusive-jet photoproduction with $E_{T}^{\mathrm{jet}}>21 \mathrm{GeV}$ in the kinematic region given by $Q^{2}<1 \mathrm{GeV}^{2}$ and $142<W_{\gamma p}<293 \mathrm{GeV}$. Other details as in the caption to Table 2.

\begin{tabular}{|c|c|c|c|c|c|c|}
\hline$\eta^{\text {jet }}$ bin & $\left\langle\eta^{\text {jet }}\right\rangle$ & $d \sigma / d \eta^{\text {jet }}(\mathrm{pb})$ & $\delta_{\text {stat }}$ & $\delta_{\text {syst }}$ & $\delta_{\mathrm{ES}}$ & $C_{\text {had }}$ \\
\hline-0.75 to -0.50 & -0.56 & 4.12 & \pm 0.13 & $\begin{array}{l}+0.55 \\
-0.49\end{array}$ & $\begin{array}{l}+0.56 \\
-0.62\end{array}$ & 0.66 \\
\hline-0.50 to -0.25 & -0.34 & 38.12 & \pm 0.60 & $\begin{array}{l}+2.51 \\
-2.45\end{array}$ & $\begin{array}{l}+3.47 \\
-3.88\end{array}$ & 0.87 \\
\hline-0.25 to +0.00 & -0.11 & 108.8 & \pm 1.1 & $\begin{array}{l}+3.8 \\
-3.8\end{array}$ & $\begin{array}{l}+7.3 \\
-7.9\end{array}$ & 0.94 \\
\hline+0.00 to +0.25 & +0.13 & 176.2 & \pm 1.4 & $\begin{array}{l}+3.8 \\
-3.6\end{array}$ & $\begin{array}{l}+9.7 \\
-10.6\end{array}$ & 0.97 \\
\hline+0.25 to +0.50 & +0.38 & 238.3 & \pm 1.7 & $\begin{array}{l}+3.3 \\
-3.2\end{array}$ & $\begin{array}{l}+11.8 \\
-12.7\end{array}$ & 0.97 \\
\hline+0.50 to +0.75 & +0.63 & 282.0 & \pm 1.8 & $\begin{array}{l}+4.6 \\
-4.4\end{array}$ & $\begin{array}{l}+13.0 \\
-14.1\end{array}$ & 0.97 \\
\hline+0.75 to +1.00 & +0.87 & 309.5 & \pm 2.0 & $\begin{array}{l}+5.9 \\
-5.9\end{array}$ & $\begin{array}{l}+14.0 \\
-14.5\end{array}$ & 0.98 \\
\hline+1.00 to +1.25 & +1.12 & 314.5 & \pm 2.0 & $\begin{array}{l}+5.1 \\
-5.1\end{array}$ & $\begin{array}{l}+13.3 \\
-14.4\end{array}$ & 0.99 \\
\hline+1.25 to +1.50 & +1.38 & 281.6 & \pm 1.8 & $\begin{array}{l}+5.9 \\
-5.9\end{array}$ & $\begin{array}{l}+11.5 \\
-12.2\end{array}$ & 1.00 \\
\hline+1.50 to +1.75 & +1.63 & 262.9 & \pm 1.7 & $\begin{array}{l}+6.5 \\
-6.5\end{array}$ & $\begin{array}{l}+11.2 \\
-11.4\end{array}$ & 1.00 \\
\hline+1.75 to +2.00 & +1.88 & 225.3 & \pm 1.6 & $\begin{array}{l}+4.3 \\
-4.3\end{array}$ & $\begin{array}{l}+9.3 \\
-9.9\end{array}$ & 1.00 \\
\hline+2.00 to +2.25 & +2.13 & 200.9 & \pm 1.5 & $\begin{array}{l}+2.6 \\
-2.6\end{array}$ & $\begin{array}{l}+8.4 \\
-9.1\end{array}$ & 1.01 \\
\hline+2.25 to +2.50 & +2.38 & 173.2 & \pm 1.3 & $\begin{array}{l}+5.3 \\
-5.3 \\
\end{array}$ & $\begin{array}{l}+7.7 \\
-8.3 \\
\end{array}$ & 1.01 \\
\hline
\end{tabular}




\section{ZEUS}
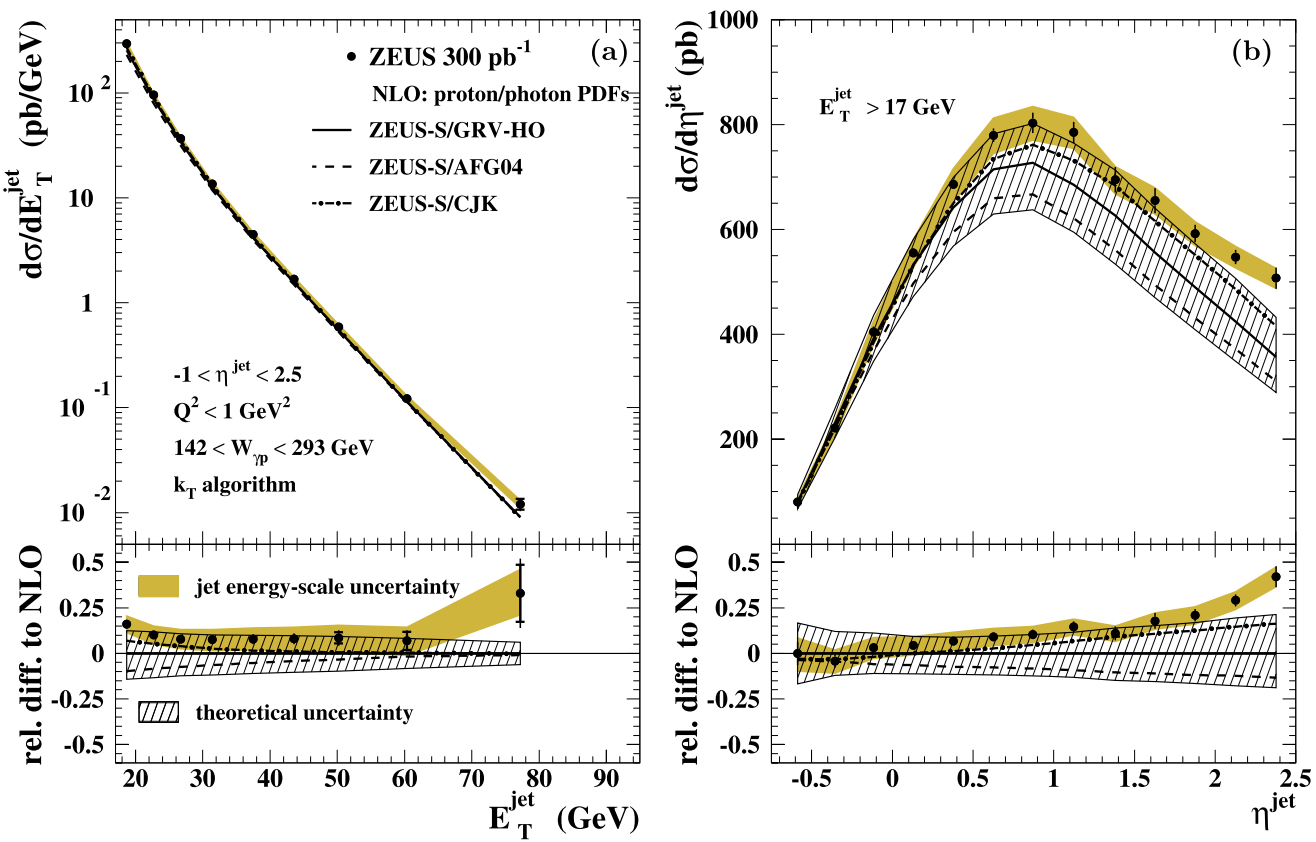

Fig. 6. The measured differential cross sections (a) $d \sigma / d E_{T}^{\text {jet }}$ and (b) $d \sigma / d \eta^{\text {jet }}$ based on the $k_{T}$ jet algorithm for inclusive-jet photoproduction with $E_{T}^{\text {jet }}>17 \mathrm{GeV}$ and $-1<\eta^{\text {jet }}<2.5$ (dots) in the kinematic region given by $Q^{2}<1 \mathrm{GeV}^{2}$ and $142<W_{\gamma p}<293 \mathrm{GeV}$. For comparison, the NLO QCD calculations using different parameterisations of the photon PDFs are also shown. Other details as in the caption to Fig. 2.

\subsection{Double-differential cross sections}

The measurements of the inclusive-jet cross sections based on the $k_{T}$ jet algorithm as functions of $E_{T}^{\text {jet }}$ in different regions of $\eta^{\text {jet }}$ are presented in Fig. 8 and Tables 4 and 5. The measured cross sections exhibit a steep fall-off within the $E_{T}^{\mathrm{jet}}$ range considered. The $E_{T}^{\mathrm{jet}}$ dependence of the cross section becomes less steep as $\eta^{\text {jet }}$ increases.

The NLO QCD predictions are compared to the measurements in Fig. 8. They give a good description of the data, except at low $E_{T}^{\text {jet }}$ and high $\eta^{\text {jet }}$. Fig. 9 shows the relative difference of the measured differential cross sections to the NLO QCD calculations. The data are well described by the predictions for $-1<\eta^{\text {jet }}<2$ in the whole $E_{T}^{\text {jet }}$ range measured. For the region $2<\eta^{\text {jet }}<2.5$, where it is observed that non-perturbative effects not related to hadronisation (see Fig. 10) might contribute significantly, the data are well described only for $E_{T}^{\mathrm{jet}}>21 \mathrm{GeV}$.

Figs. 11 and 12 show the comparison between the measured cross sections and the predictions based on different photon and proton PDFs, respectively. As discussed above, differences at low $E_{T}^{\mathrm{jet}}$ and high $\eta^{\text {jet }}$ are observed between the predictions based on GRV-HO, AFG04 and CJK. The latter gives predictions closest to the data, especially in the region $2<\eta^{\text {jet }}<2.5$. The largest differences between the predictions based on MSTW08 and ZEUS-S are observed at high $E_{T}^{\text {jet }}$ 


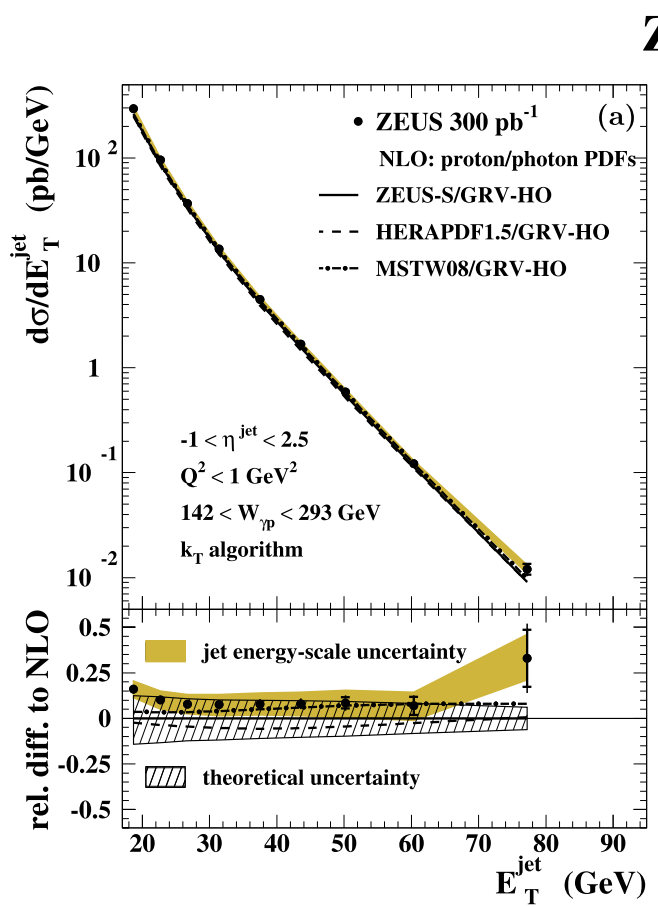

\section{ZEUS}

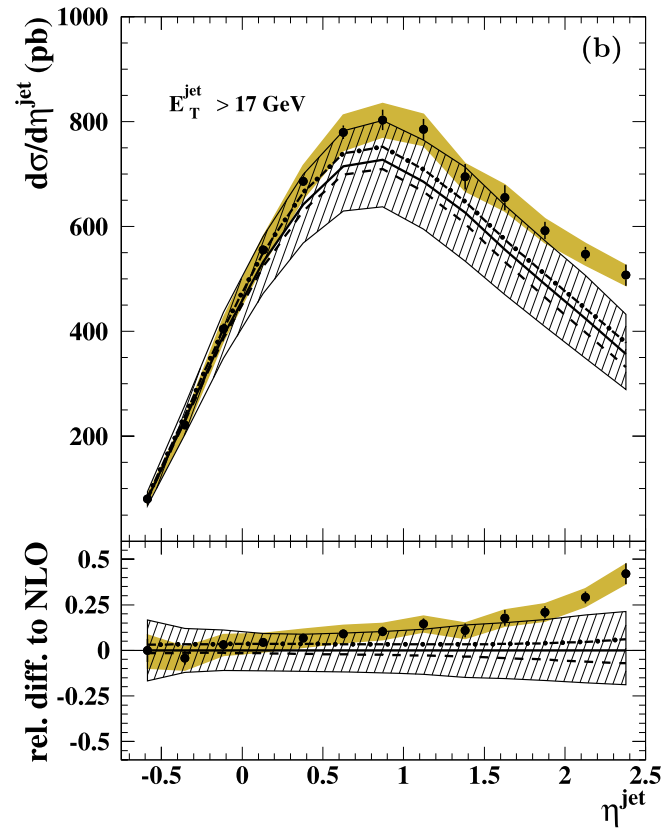

Fig. 7. The measured differential cross sections (a) $d \sigma / d E_{T}^{\text {jet }}$ and (b) $d \sigma / d \eta^{\text {jet }}$ based on the $k_{T}$ jet algorithm for inclusive-jet photoproduction with $E_{T}^{\text {jet }}>17 \mathrm{GeV}$ and $-1<\eta^{\text {jet }}<2.5$ (dots) in the kinematic region given by $Q^{2}<1 \mathrm{GeV}^{2}$ and $142<W_{\gamma p}<293 \mathrm{GeV}$. For comparison, the NLO QCD calculations using different parameterisations of the proton PDFs are also shown. Other details as in the caption to Fig. 2.

for $\eta^{\text {jet }}>1$. The predictions based on HERAPDF1.5 are lower than those based on ZEUS-S in most of the phase-space region.

As discussed in Section 8, the theoretical uncertainties are dominated by the contribution from higher orders. This uncertainty decreases as $E_{T}^{\mathrm{jet}}$ increases. The contribution from the proton PDF uncertainty is significant and approximately constant for $E_{T}^{\text {jet }}>30 \mathrm{GeV}$; at high $E_{T}^{\text {jet }}$ values, the proton PDF uncertainty is of the same order as that coming from higher orders. In these regions, in which the gluon-induced contribution is still substantial and the possible presence of nonperturbative effects is expected to be minimised, the data have the potential to constrain the gluon density in the proton. The uncertainty coming from the photon PDFs is largest at low $E_{T}^{\mathrm{jet}}$ and high $\eta^{\text {jet }}$ and approximately of the same order as that coming from higher-order terms. Therefore, these high-precision measurements also have the potential to constrain the photon PDFs in these regions of phase space.

\subsection{Single-differential cross sections based on different jet algorithms}

The measurements of the inclusive-jet cross sections based on the anti- $k_{T}$ and SIScone jet algorithms as functions of $E_{T}^{\mathrm{jet}}$ and $\eta^{\mathrm{jet}}$ are presented in Fig. 13, together with those based on the $k_{T}$ algorithm. The measured cross sections are also given in Tables 6 and 7 . The measured 


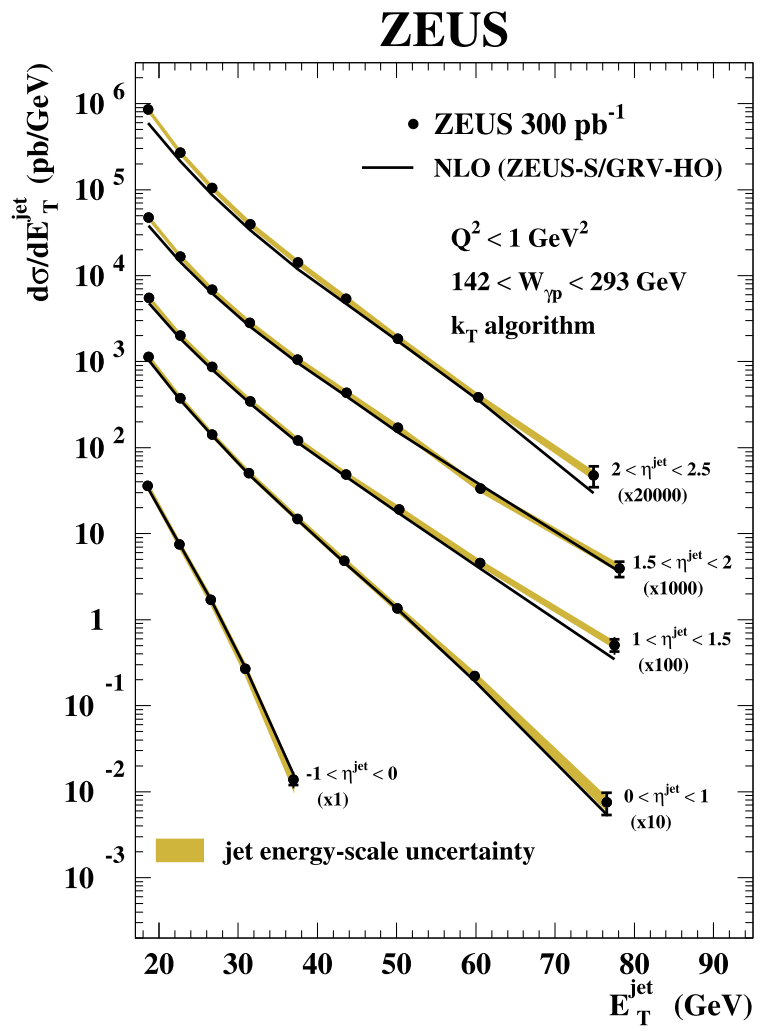

Fig. 8. The measured differential cross-sections $d \sigma / d E_{T}^{\text {jet }}$ based on the $k_{T}$ jet algorithm for inclusive-jet photoproduction with $E_{T}^{\text {jet }}>17 \mathrm{GeV}$ in different regions of $\eta^{\text {jet }}$ (dots) in the kinematic region given by $Q^{2}<1 \mathrm{GeV}^{2}$ and $142<W_{\gamma p}<$ $293 \mathrm{GeV}$. Each cross section has been multiplied by the scale factor indicated in brackets to aid visibility. Other details as in the caption to Fig. 2.

$d \sigma / d E_{T}^{\mathrm{jet}}$ cross sections exhibit a steep fall-off of over four orders of magnitude in the $E_{T}^{\mathrm{jet}}$ measured range. The measured $d \sigma / d \eta^{\text {jet }}$ cross sections display a maximum around $\eta^{\text {jet }} \approx 1$. The measured cross sections using the three jet algorithms have a similar shape, normalisation and precision.

The NLO QCD predictions are compared to the data in Fig. 13. The hadronisation correction factors applied to the calculations and their uncertainties are also shown. It is seen that the hadronisation correction factors are closest to (farthest from) unity for the $k_{T}$ (SIScone) jet algorithm (see also Tables 6 and 7). The ratios of the measured cross sections to the NLO QCD calculations are shown in Fig. 14 separately for each jet algorithm. The measured cross sections are well reproduced by the calculations, except at high $\eta^{\text {jet }}$.

The ratios of the cross sections anti- $k_{T} / k_{T}$, SIScone $/ k_{T}$ and anti- $k_{T} /$ SIScone were studied to compare the jet algorithms in more detail. These ratios allow, in particular, a stringent test of the description of the differences between jet algorithms in terms of parton radiation due to the partial cancellation of experimental and theoretical uncertainties. The measured ratios are shown in Fig. 15. In these ratios, the statistical correlations among the event samples as well as those among the jets in the same event were taken into account in the estimation of the statistical uncertainties; most of the systematic uncertainties, including that due to the jet energy scale, 


\section{RAPID COMMUNICATION}

Table 4

The measured differential cross sections $d \sigma / d E_{T}^{\text {jet }}$ based on the $k_{T}$ jet algorithm for inclusive-jet photoproduction with $E_{T}^{\text {jet }}>17 \mathrm{GeV}$ in different regions of $\eta^{\text {jet }}$ in the kinematic region given by $Q^{2}<1 \mathrm{GeV}^{2}$ and $142<W_{\gamma p}<293 \mathrm{GeV}$. Other details as in the caption to Table 2.

\begin{tabular}{|c|c|c|c|c|c|c|}
\hline$E_{T}^{\mathrm{jet}}$ bin $(\mathrm{GeV})$ & $\left\langle E_{T}^{\mathrm{jet}}\right\rangle(\mathrm{GeV})$ & $d \sigma / d E_{T}^{\mathrm{jet}}(\mathrm{pb} / \mathrm{GeV})$ & $\delta_{\text {stat }}$ & $\delta_{\text {syst }}$ & $\delta_{\mathrm{ES}}$ & $C_{\text {had }}$ \\
\hline \multicolumn{7}{|l|}{$-1<\eta^{\text {jet }}<0$} \\
\hline $17-21$ & 18.6 & 35.99 & \pm 0.17 & $\begin{array}{l}+1.15 \\
-1.14\end{array}$ & $\begin{array}{l}+2.04 \\
-2.21\end{array}$ & 0.93 \\
\hline $21-25$ & 22.6 & 7.522 & \pm 0.071 & $\begin{array}{l}+0.337 \\
-0.331\end{array}$ & $\begin{array}{l}+0.543 \\
-0.592\end{array}$ & 0.91 \\
\hline $25-29$ & 26.5 & 1.695 & \pm 0.032 & $\begin{array}{l}+0.115 \\
-0.109\end{array}$ & $\begin{array}{l}+0.157 \\
-0.167\end{array}$ & 0.89 \\
\hline $29-35$ & 30.9 & 0.268 & \pm 0.010 & $\begin{array}{l}+0.024 \\
-0.024\end{array}$ & $\begin{array}{l}+0.032 \\
-0.039\end{array}$ & 0.85 \\
\hline $35-41$ & 37.0 & 0.0138 & \pm 0.0018 & $\begin{array}{l}+0.0026 \\
-0.0021\end{array}$ & $\begin{array}{l}+0.0023 \\
-0.0031\end{array}$ & 0.80 \\
\hline \multicolumn{7}{|l|}{$0<\eta^{\text {jet }}<1$} \\
\hline $17-21$ & 18.7 & 113.61 & \pm 0.31 & $\begin{array}{l}+1.68 \\
-1.67\end{array}$ & $\begin{array}{l}+4.45 \\
-4.72\end{array}$ & 0.99 \\
\hline $21-25$ & 22.7 & 37.73 & \pm 0.17 & $\begin{array}{l}+0.54 \\
-0.53\end{array}$ & $\begin{array}{l}+1.70 \\
-1.83\end{array}$ & 0.98 \\
\hline $25-29$ & 26.7 & 14.27 & \pm 0.11 & $\begin{array}{l}+0.24 \\
-0.24\end{array}$ & $\begin{array}{l}+0.72 \\
-0.74\end{array}$ & 0.98 \\
\hline $29-35$ & 31.4 & 5.034 & \pm 0.052 & $\begin{array}{l}+0.093 \\
-0.087\end{array}$ & $\begin{array}{l}+0.261 \\
-0.300\end{array}$ & 0.97 \\
\hline $35-41$ & 37.5 & 1.490 & \pm 0.027 & $\begin{array}{l}+0.040 \\
-0.039\end{array}$ & $\begin{array}{l}+0.090 \\
-0.098\end{array}$ & 0.96 \\
\hline $41-47$ & 43.4 & 0.485 & \pm 0.015 & $\begin{array}{l}+0.016 \\
-0.015\end{array}$ & $\begin{array}{l}+0.031 \\
-0.032\end{array}$ & 0.96 \\
\hline $47-55$ & 50.1 & 0.1356 & \pm 0.0068 & $\begin{array}{l}+0.0059 \\
-0.0056\end{array}$ & $\begin{array}{l}+0.0099 \\
-0.0110\end{array}$ & 0.95 \\
\hline $55-71$ & 59.8 & 0.0220 & \pm 0.0017 & $\begin{array}{l}+0.0014 \\
-0.0013\end{array}$ & $\begin{array}{l}+0.0019 \\
-0.0019\end{array}$ & 0.93 \\
\hline 71-95 & 76.5 & 0.00075 & \pm 0.00022 & $\begin{array}{l}+0.00011 \\
-0.00008\end{array}$ & $\begin{array}{l}+0.00010 \\
-0.00015\end{array}$ & 0.93 \\
\hline \multicolumn{7}{|l|}{$1<\eta^{\text {jet }}<1.5$} \\
\hline $17-21$ & 18.7 & 55.26 & \pm 0.21 & $\begin{array}{l}+2.07 \\
-2.07\end{array}$ & $\begin{array}{l}+1.81 \\
-1.92\end{array}$ & 1.00 \\
\hline $21-25$ & 22.7 & 20.17 & \pm 0.12 & $\begin{array}{l}+0.43 \\
-0.43\end{array}$ & $\begin{array}{l}+0.77 \\
-0.83\end{array}$ & 1.00 \\
\hline $25-29$ & 26.7 & 8.723 & \pm 0.082 & $\begin{array}{l}+0.133 \\
-0.133\end{array}$ & $\begin{array}{l}+0.355 \\
-0.387\end{array}$ & 0.99 \\
\hline $29-35$ & 31.5 & 3.461 & \pm 0.044 & $\begin{array}{l}+0.062 \\
-0.060\end{array}$ & $\begin{array}{l}+0.173 \\
-0.184\end{array}$ & 0.99 \\
\hline $35-41$ & 37.6 & 1.209 & \pm 0.026 & $\begin{array}{l}+0.031 \\
-0.031\end{array}$ & $\begin{array}{l}+0.060 \\
-0.058\end{array}$ & 0.97 \\
\hline $41-47$ & 43.6 & 0.486 & \pm 0.016 & $\begin{array}{l}+0.013 \\
-0.013\end{array}$ & $\begin{array}{l}+0.026 \\
-0.027\end{array}$ & 0.99 \\
\hline $47-55$ & 50.3 & 0.1911 & \pm 0.0089 & $\begin{array}{l}+0.0074 \\
-0.0073\end{array}$ & $\begin{array}{l}+0.0117 \\
-0.0130\end{array}$ & 0.97 \\
\hline $55-71$ & 60.5 & 0.0454 & \pm 0.0031 & $\begin{array}{l}+0.0023 \\
-0.0023\end{array}$ & $\begin{array}{l}+0.0028 \\
-0.0031\end{array}$ & 0.96 \\
\hline $71-95$ & 77.5 & 0.00508 & \pm 0.00083 & $\begin{array}{l}+0.00083 \\
-0.00083 \\
\end{array}$ & $\begin{array}{l}+0.00041 \\
-0.00038 \\
\end{array}$ & 0.99 \\
\hline
\end{tabular}

cancel out. The measurements show that the cross sections based on the anti- $k_{T}$ algorithm are similar in shape to those based on the $k_{T}$ algorithm but $\approx 6 \%$ lower and that the cross sections based on the SIScone have a slightly different shape than those based on the $k_{T}$ and anti- $k_{T}$ algorithms. 
Table 5

Continuation of Table 4.

\begin{tabular}{|c|c|c|c|c|c|c|}
\hline$E_{T}^{\mathrm{jet}} \operatorname{bin}(\mathrm{GeV})$ & $\left\langle E_{T}^{\mathrm{jet}}\right\rangle(\mathrm{GeV})$ & $d \sigma / d E_{T}^{\mathrm{jet}}(\mathrm{pb} / \mathrm{GeV})$ & $\delta_{\text {stat }}$ & $\delta_{\text {syst }}$ & $\delta_{\mathrm{ES}}$ & $C_{\text {had }}$ \\
\hline \multicolumn{7}{|l|}{$1.5<\eta^{\text {jet }}<2$} \\
\hline $17-21$ & 18.7 & 47.51 & \pm 0.19 & $\begin{array}{l}+1.86 \\
-1.87\end{array}$ & $\begin{array}{l}+1.64 \\
-1.68\end{array}$ & 0.99 \\
\hline $21-25$ & 22.7 & 16.70 & \pm 0.11 & $\begin{array}{l}+0.48 \\
-0.48\end{array}$ & $\begin{array}{l}+0.66 \\
-0.68\end{array}$ & 1.00 \\
\hline $25-29$ & 26.7 & 6.844 & \pm 0.069 & $\begin{array}{l}+0.134 \\
-0.134\end{array}$ & $\begin{array}{l}+0.285 \\
-0.294\end{array}$ & 0.99 \\
\hline $29-35$ & 31.5 & 2.818 & \pm 0.037 & $\begin{array}{l}+0.035 \\
-0.035\end{array}$ & $\begin{array}{l}+0.129 \\
-0.138\end{array}$ & 0.99 \\
\hline $35-41$ & 37.5 & 1.055 & \pm 0.023 & $\begin{array}{l}+0.020 \\
-0.020\end{array}$ & $\begin{array}{l}+0.056 \\
-0.052\end{array}$ & 0.99 \\
\hline $41-47$ & 43.7 & 0.432 & \pm 0.015 & $\begin{array}{l}+0.006 \\
-0.006\end{array}$ & $\begin{array}{l}+0.023 \\
-0.026\end{array}$ & 0.99 \\
\hline $47-55$ & 50.2 & 0.1703 & \pm 0.0086 & $\begin{array}{l}+0.0020 \\
-0.0022\end{array}$ & $\begin{array}{l}+0.0099 \\
-0.0119\end{array}$ & 0.99 \\
\hline $55-71$ & 60.6 & 0.0334 & \pm 0.0027 & $\begin{array}{l}+0.0012 \\
-0.0011\end{array}$ & $\begin{array}{l}+0.0021 \\
-0.0025\end{array}$ & 1.00 \\
\hline $71-95$ & 78.1 & 0.00393 & \pm 0.00081 & $\begin{array}{l}+0.00027 \\
-0.00028\end{array}$ & $\begin{array}{l}+0.00041 \\
-0.00029\end{array}$ & 0.98 \\
\hline \multicolumn{7}{|l|}{$2<\eta^{\text {jet }}<2.5$} \\
\hline $17-21$ & 18.6 & 42.88 & \pm 0.18 & $\begin{array}{l}+1.63 \\
-1.63\end{array}$ & $\begin{array}{l}+1.40 \\
-1.43\end{array}$ & 1.00 \\
\hline $21-25$ & 22.7 & 13.479 & \pm 0.097 & $\begin{array}{l}+0.325 \\
-0.325\end{array}$ & $\begin{array}{l}+0.523 \\
-0.591\end{array}$ & 1.01 \\
\hline $25-29$ & 26.7 & 5.223 & \pm 0.058 & $\begin{array}{l}+0.134 \\
-0.134\end{array}$ & $\begin{array}{l}+0.240 \\
-0.222\end{array}$ & 1.01 \\
\hline $29-35$ & 31.5 & 1.977 & \pm 0.029 & $\begin{array}{l}+0.027 \\
-0.027\end{array}$ & $\begin{array}{l}+0.091 \\
-0.114\end{array}$ & 1.00 \\
\hline $35-41$ & 37.6 & 0.708 & \pm 0.018 & $\begin{array}{l}+0.016 \\
-0.015\end{array}$ & $\begin{array}{l}+0.039 \\
-0.038\end{array}$ & 1.00 \\
\hline $41-47$ & 43.6 & 0.268 & \pm 0.011 & $\begin{array}{l}+0.003 \\
-0.004\end{array}$ & $\begin{array}{l}+0.017 \\
-0.017\end{array}$ & 1.00 \\
\hline $47-55$ & 50.2 & 0.0928 & \pm 0.0059 & $\begin{array}{l}+0.0065 \\
-0.0065\end{array}$ & $\begin{array}{l}+0.0061 \\
-0.0082\end{array}$ & 1.00 \\
\hline $55-71$ & 60.3 & 0.0192 & \pm 0.0019 & $\begin{array}{l}+0.0007 \\
-0.0007\end{array}$ & $\begin{array}{l}+0.0016 \\
-0.0013\end{array}$ & 0.97 \\
\hline 71-95 & 74.9 & 0.00238 & \pm 0.00065 & $\begin{array}{l}+0.00023 \\
-0.00023 \\
\end{array}$ & $\begin{array}{l}+0.00024 \\
-0.00021 \\
\end{array}$ & 1.11 \\
\hline
\end{tabular}

The pQCD predictions including up to $\mathcal{O}\left(\alpha_{s}^{2}\right)$ terms for the ratios of the cross sections are also shown in Fig. 15. In the estimation of the total theoretical uncertainty of the predicted ratios, all the theoretical contributions were assumed to be correlated except those due to terms beyond $\mathcal{O}\left(\alpha_{s}^{2}\right)$ and to the modelling of the QCD cascade and hadronisation. Fig. 15 also includes the ratio of the hadronisation correction factors applied to the $\mathcal{O}\left(\alpha_{s}^{2}\right)$ predictions. The predictions for the anti- $k_{T}$ and $k_{T}$ algorithms are identical at $\mathcal{O}\left(\alpha_{s}^{2}\right)$ and, thus, the predicted anti- $k_{T} / k_{T}$ ratio coincides with the ratio of the hadronisation corrections for both algorithms. The predictions for the $k_{T}$ and anti- $k_{T}$ algorithms are expected to start to differ at $\mathcal{O}\left(\alpha_{s}^{3}\right)$ and, conservatively, the uncertainties due to higher-order terms were assumed to be uncorrelated in the ratio; otherwise, a coherent variation of the renormalisation scale in the ratio would yield an unrealistic zero contribution. In the case of the SIScone $/ k_{T}$ and anti- $k_{T} / \mathrm{SIScone}$, the $\mathcal{O}\left(\alpha_{s}^{2}\right)$ predictions are different and the ratios differ significantly from the ratios of the hadronisation correction factors. The measured ratios are well described by the calculations including terms up to $\mathcal{O}\left(\alpha_{s}^{2}\right)$ within 


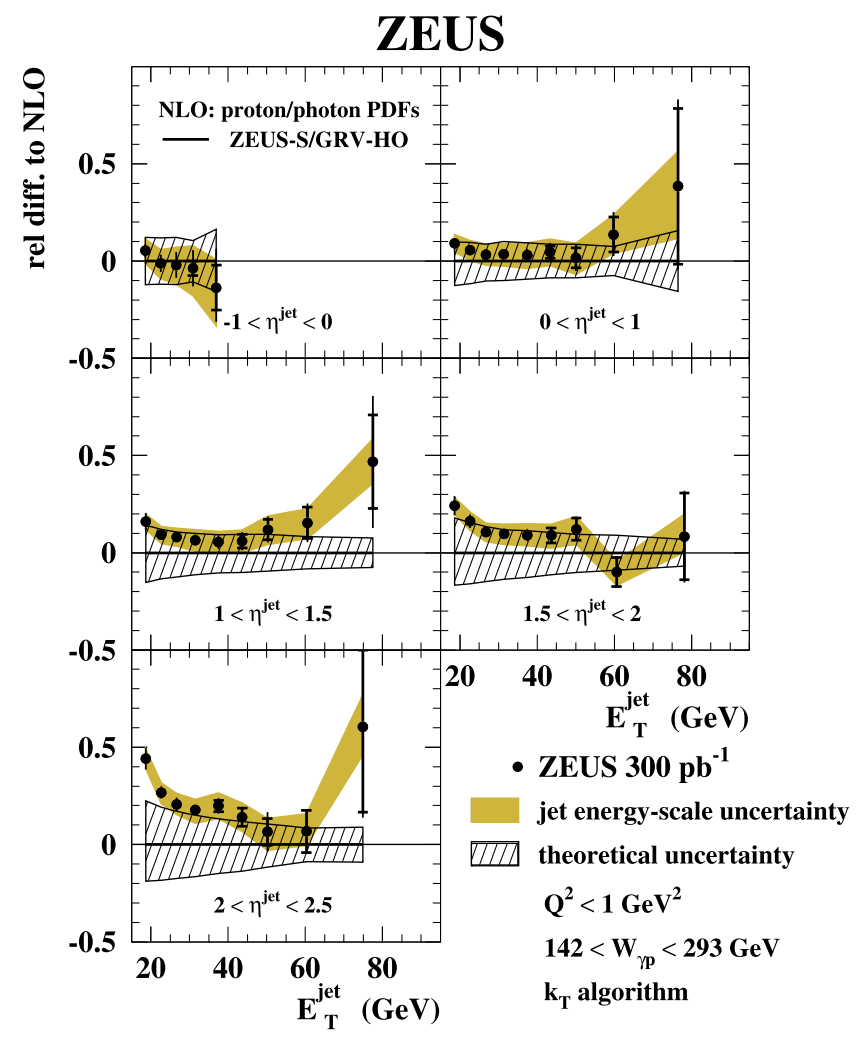

Fig. 9. The relative differences between the measured differential cross sections $d \sigma / d E_{T}^{\text {jet }}$ presented in Fig. 8 and the NLO QCD calculations (dots). Other details as in the caption to Fig. 2.

the experimental and theoretical uncertainties, which are dominated by the uncertainty due to higher orders.

The differences in the influence of non-perturbative effects not related to hadronisation for the different jet algorithms were investigated by comparing the ratios of the NLO $\otimes$ NP predictions with $p_{T \text {, min }}^{\mathrm{sec}}=1.5 \mathrm{GeV}$, which are also shown in Fig. 15 . For the anti- $k_{T} / k_{T}$ ratio, the NLO and the NLO $\otimes N P$ predictions are very similar, which indicates that the non-perturbative effects affect the jets in the same way. However, in the SIScone $k_{T}$ and anti- $k_{T} /$ SIScone ratios, the ratios of the NLO $\otimes N P$ predictions differ from the ratios of the NLO calculations at low $E_{T}^{\text {jet }}$ and high $\eta^{\text {jet}}$; these differences, which are located in the regions of phase space where the NLO calculations fail to describe the data, show that there is a dependence on the details of the jet reconstruction concerning non-perturbative effects not related to hadronisation. These differences are at most of the same size as the theoretical uncertainties.

In summary, it is concluded that the measured inclusive-jet cross sections based on the $k_{T}$, anti- $k_{T}$ and SIScone jet algorithms are well described by the NLO QCD calculations, except at high $\eta^{\text {jet }}$. The data and the calculations for the three jet algorithms have a similar experimental and theoretical precision. Furthermore, the measured ratios are well described by the predictions including up to $\mathcal{O}\left(\alpha_{s}^{2}\right)$ terms, demonstrating the ability of the pQCD calculations with up to three 


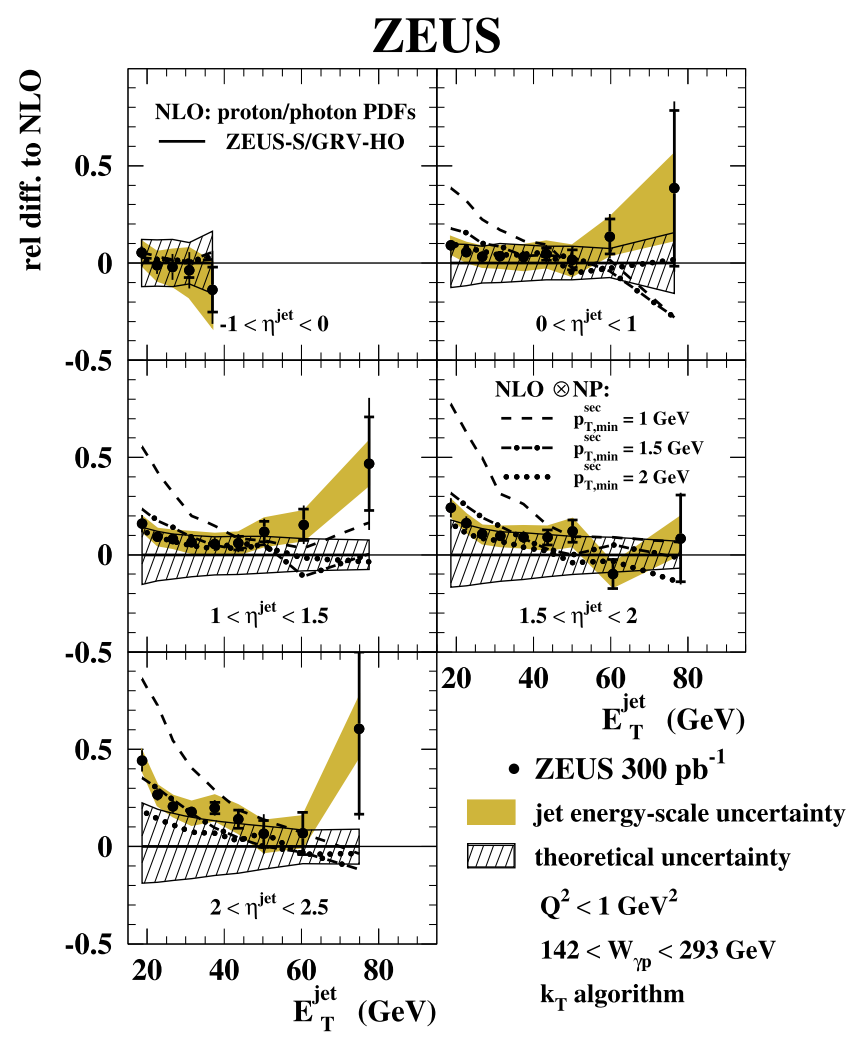

Fig. 10. The relative differences between the measured differential cross sections $d \sigma / d E_{T}^{\text {jet }}$ presented in Fig. 8 and the NLO QCD calculations (dots). The relative differences between the predictions based on the calculations including an estimation of non-perturbative effects (see text) and the NLO QCD calculation are also shown. Other details as in the caption to Fig. 2.

partons in the final state to account adequately for the differences between the SIScone and the $k_{T}$ or anti- $k_{T}$ jet algorithms.

\subsection{Determination of $\alpha_{S}\left(M_{Z}\right)$}

The measured single-differential cross sections $d \sigma / d E_{T}^{\mathrm{jet}}$ based on the three jet algorithms were used to determine values of $\alpha_{S}\left(M_{Z}\right)$ using the method presented previously [2]. The NLO QCD calculations were performed using five different sets of the ZEUS-S proton PDFs which were determined from global fits assuming different values of $\alpha_{S}\left(M_{Z}\right)$, namely $\alpha_{S}\left(M_{Z}\right)=0.115$, $0.117,0.119,0.121$ and 0.123 . The GRV-HO set was used as default for the photon PDFs. The value of $\alpha_{S}\left(M_{Z}\right)$ used in each calculation was that associated with the corresponding set of proton PDFs.

The $\alpha_{S}\left(M_{Z}\right)$ dependence of the predicted cross sections in each bin $i$ of $E_{T}^{\mathrm{jet}}$ was parameterised according to

$$
\left[d \sigma / d E_{T}^{\mathrm{jet}}\left(\alpha_{s}\left(M_{Z}\right)\right)\right]_{i}=C_{1}^{i} \alpha_{s}\left(M_{Z}\right)+C_{2}^{i} \alpha_{s}^{2}\left(M_{Z}\right),
$$




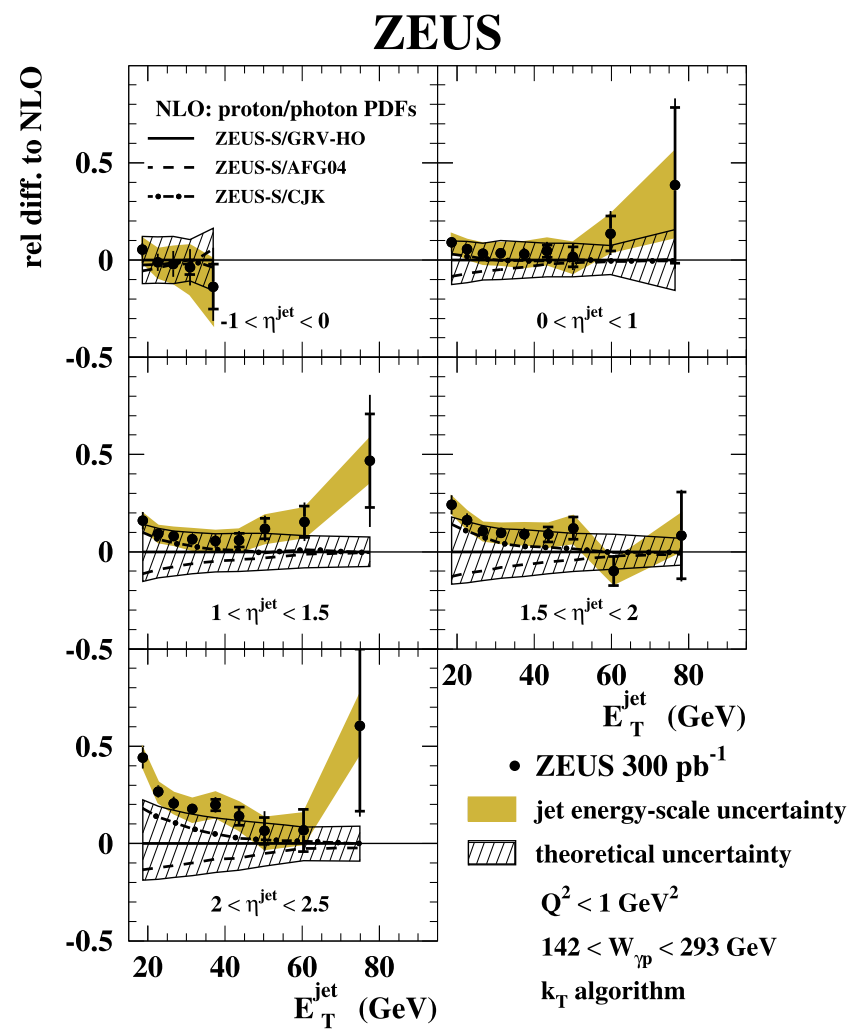

Fig. 11. The relative differences between the measured differential cross sections $d \sigma / d E_{T}^{\text {jet }}$ presented in Fig. 8 and the NLO QCD calculations (dots). The relative differences between the predictions based on different photon PDFs and that based on the ZEUS-S/GRV-HO sets are also shown. Other details as in the caption to Fig. 2.

where $C_{1}^{i}$ and $C_{2}^{i}$ were determined from a $\chi^{2}$ fit to the NLO QCD calculations. The value of $\alpha_{S}\left(M_{Z}\right)$ was determined by a $\chi^{2}$ fit to the measured $d \sigma / d E_{T}^{\mathrm{jet}}$ values. In the fitting procedure, the running of $\alpha_{s}$ as predicted by QCD was assumed. Only the measurements for $E_{T}^{\text {jet }}>21 \mathrm{GeV}$ were used in the fit to minimise the effects of a possible non-perturbative contribution in addition to that of hadronisation and the uncertainty coming from higher orders. In addition, the fit was restricted to $E_{T}^{\text {jet }}<71 \mathrm{GeV}$ because of the relatively large uncertainty coming from the proton PDFs for higher $E_{T}^{\text {jet }}$ values.

The experimental uncertainties on the extracted values of $\alpha_{S}\left(M_{Z}\right)$ were evaluated by repeating the analysis for each systematic check presented in Section 7. The overall normalisation uncertainty from the luminosity determination was also included. The largest contribution comes from the uncertainty in the absolute jet energy scale. The theoretical uncertainties were evaluated as described in Section 8. The largest contribution arises from the terms beyond NLO, which was estimated by using the method of Jones et al. [68]. The uncertainty due to the photon PDFs is of the same order as that arising from higher orders. The uncertainty due to the proton PDFs and that arising from the hadronisation effects were also estimated. All uncertainties are listed separately in Table 8. 


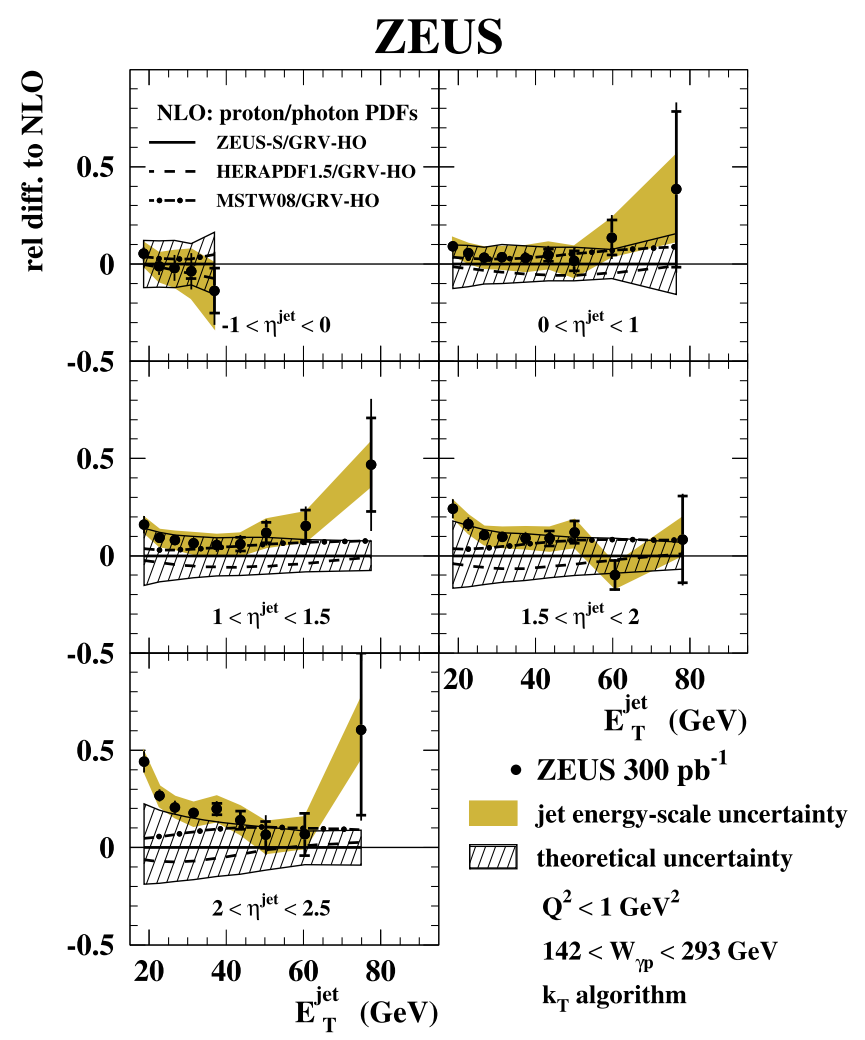

Fig. 12. The relative differences between the measured differential cross sections $d \sigma / d E_{T}^{\text {jet }}$ presented in Fig. 8 and the NLO QCD calculations (dots). The relative differences between the predictions based on different proton PDFs and that based on the ZEUS-S/GRV-HO sets are also shown. Other details as in the caption to Fig. 2.

As a cross-check, $\alpha_{S}\left(M_{Z}\right)$ was determined by using NLO QCD calculations based on the CTEQ6.1 [69] (MSTW08) sets of proton PDFs. The values obtained are consistent within 1.0 (1.0), $0.9(0.9)$ and $0.6(0.8) \%$ with those based on ZEUS-S for the $k_{T}$, anti- $k_{T}$ and SIScone determinations, respectively. The uncertainty arising from the proton PDFs was estimated to be $\pm 1.3(0.9,0.5) \%$ for the $k_{T}$ and anti- $k_{T}$ and $\pm 1.2(0.8,0.4) \%$ for the SIScone determinations using the results of the CTEQ6.1 (MSTW08nlo90cl, MSTW08nlo68cl) analysis.

The values of $\alpha_{S}\left(M_{Z}\right)$ obtained from the measured $d \sigma / d E_{T}^{\text {jet }}$ are

$$
\begin{aligned}
& \left.\alpha_{S}\left(M_{Z}\right)\right|_{k_{T}}=0.1206_{-0.0022}^{+0.0023} \text { (exp.) }{ }_{-0.0035}^{+0.0042} \text { (th.), } \\
& \left.\left.\alpha_{S}\left(M_{Z}\right)\right|_{\text {anti }-k_{T}}=0.1198_{-0.0022}^{+0.0023} \text { (exp. }\right)_{-0.0034}^{+0.0041} \text { (th.), } \\
& \left.\alpha_{S}\left(M_{Z}\right)\right|_{\text {SIScone }}=0.1196_{-0.0021}^{+0.0022} \text { (exp.) }{ }_{-0.0043}^{+0.0046} \text { (th.). }
\end{aligned}
$$

The value of $\alpha_{S}\left(M_{Z}\right)$ determined from the anti- $k_{T}$ (SIScone) measurements is consistent with that obtained from the $k_{T}$ analysis within $-0.7(-0.8) \%$. The total uncertainty from each determination, as shown in Table 8, indicates that the performance of the three jet algorithms is similar. These determinations are consistent with previous determinations in NC DIS [5-7] at HERA, with the results obtained in $p \bar{p}$ collisions [70] and have a precision comparable to those obtained in individual determinations from $e^{+} e^{-}$experiments [71]. These values are also con- 


\section{ZEUS}
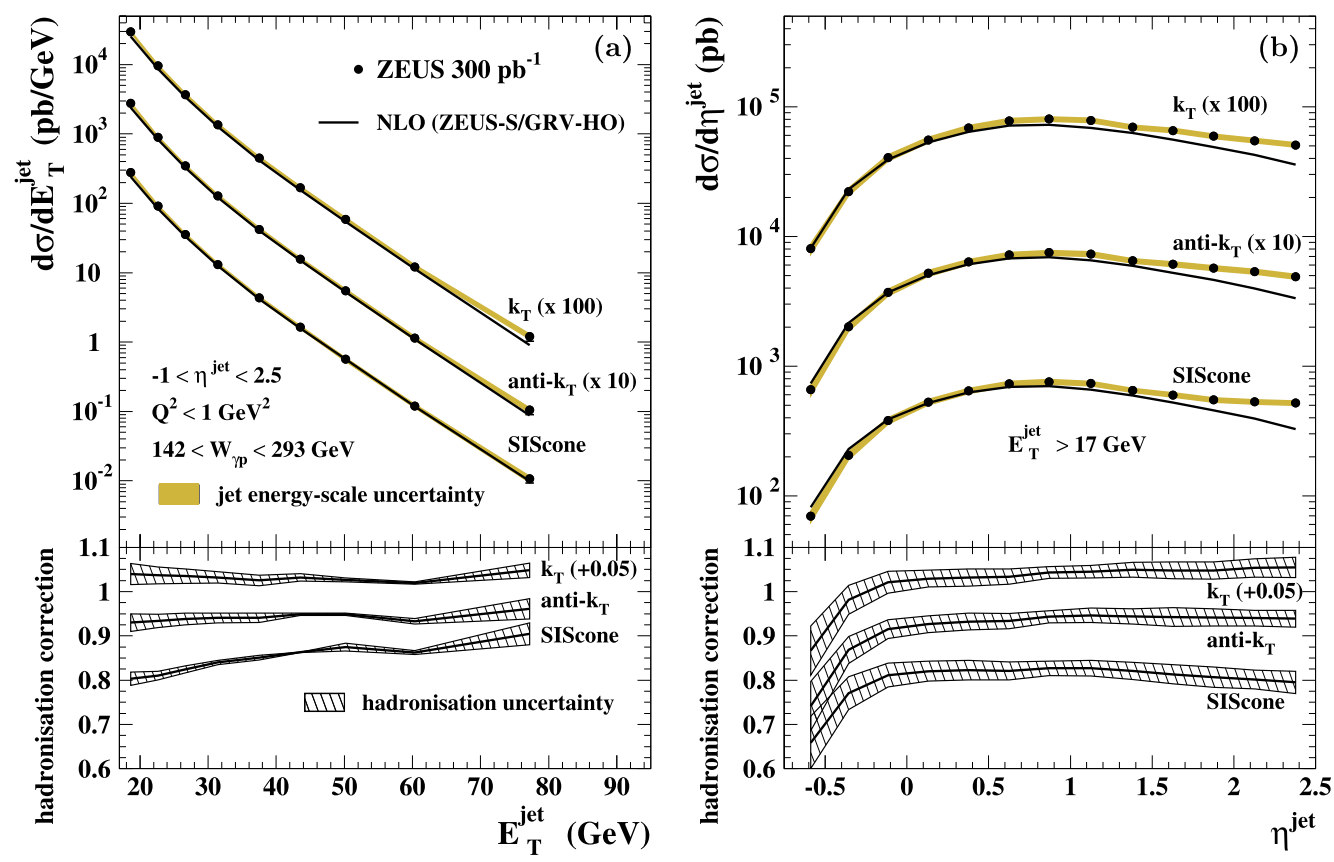

Fig. 13. The measured differential cross sections (a) $d \sigma / d E_{T}^{\text {jet }}$ and (b) $d \sigma / d \eta^{\text {jet }}$ based on different jet algorithms for inclusive-jet photoproduction with $E_{T}^{\text {jet }}>17 \mathrm{GeV}$ and $-1<\eta^{\text {jet }}<2.5$ (dots) in the kinematic region given by $Q^{2}<$ $1 \mathrm{GeV}^{2}$ and $142<W_{\gamma p}<293 \mathrm{GeV}$. The anti- $k_{T}$ and $k_{T}$ cross sections were multiplied by the scale factors indicated in brackets to aid visibility. The lower part of the figure shows the hadronisation correction factors applied to the NLO calculations together with their uncertainty (hatched bands) for each jet algorithm; the hadronisation correction factor for the $k_{T}$ algorithm was shifted by the value indicated in brackets to aid visibility. Other details as in the caption to Fig. 2.

sistent with the world average [63], as well as with the HERA 2004 average [72] and the HERA 2007 combined value [73]. Fig. 16 shows the value of $\alpha_{S}\left(M_{Z}\right)$ determined from the $k_{T}$-based analysis together with determinations from other experiments and the HERA and world averages.

\subsection{Energy-scale dependence of $\alpha_{s}$}

The energy-scale dependence of $\alpha_{s}$ was determined from an NLO QCD fit to the measured $d \sigma / d E_{T}^{\text {jet }}$ cross section. Values of $\alpha_{s}$ were extracted at each mean value of measured $E_{T}^{\text {jet }}$ without assuming the running of $\alpha_{s}$. The method employed was the same as described above, but parameterising the $\alpha_{s}$ dependence of $d \sigma / d E_{T}^{\text {jet }}$ in terms of $\alpha_{S}\left(\left\langle E_{T}^{\text {jet }}\right\rangle\right)$ instead of $\alpha_{s}\left(M_{Z}\right)$, where $\left\langle E_{T}^{\mathrm{jet}}\right\rangle$ is the average $E_{T}^{\mathrm{jet}}$ of the data in each bin.

The extracted values of $\alpha_{s}$ as a function of $E_{T}^{\text {jet }}$ are shown in Fig. 17 and Table 9 for the analysis based on the $k_{T}$ algorithm. The data demonstrate the running of $\alpha_{s}$ over a large range in $E_{T}^{\text {jet }}$ from a single experiment. The predicted running of the strong coupling [74] calculated at two loops is in good agreement with the data. The values of $\alpha_{s}$ as functions of $E_{T}^{\mathrm{jet}}$ determined 
Table 6

The measured differential cross sections $d \sigma / d E_{T}^{\text {jet }}$ based on different jet algorithms for inclusive-jet photoproduction with $-1<\eta^{\text {jet }}<2.5$ in the kinematic region given by $Q^{2}<1 \mathrm{GeV}^{2}$ and $142<W_{\gamma p}<293 \mathrm{GeV}$. Other details as in the caption to Table 2 .

\begin{tabular}{|c|c|c|c|c|c|c|}
\hline$E_{T}^{\mathrm{jet}} \operatorname{bin}(\mathrm{GeV})$ & $\left\langle E_{T}^{\mathrm{jet}}\right\rangle(\mathrm{GeV})$ & $d \sigma / d E_{T}^{\mathrm{jet}}(\mathrm{pb} / \mathrm{GeV})$ & $\delta_{\text {stat }}$ & $\delta_{\text {syst }}$ & $\delta_{\mathrm{ES}}$ & $C_{\text {had }}$ \\
\hline \multicolumn{7}{|l|}{ anti- $_{T}$} \\
\hline $17-21$ & 18.6 & 276.84 & \pm 0.50 & $\begin{array}{l}+5.55 \\
-5.55\end{array}$ & $\begin{array}{l}+11.09 \\
-11.75\end{array}$ & 0.93 \\
\hline $21-25$ & 22.7 & 89.51 & \pm 0.28 & $\begin{array}{l}+1.36 \\
-1.35\end{array}$ & $\begin{array}{l}+4.03 \\
-4.30\end{array}$ & 0.93 \\
\hline $25-29$ & 26.7 & 34.54 & \pm 0.18 & $\begin{array}{l}+0.49 \\
-0.49\end{array}$ & $\begin{array}{l}+1.68 \\
-1.76\end{array}$ & 0.94 \\
\hline $29-35$ & 31.4 & 12.771 & \pm 0.091 & $\begin{array}{l}+0.180 \\
-0.170\end{array}$ & $\begin{array}{l}+0.640 \\
-0.714\end{array}$ & 0.94 \\
\hline $35-41$ & 37.5 & 4.218 & \pm 0.052 & $\begin{array}{l}+0.090 \\
-0.087\end{array}$ & $\begin{array}{l}+0.235 \\
-0.243\end{array}$ & 0.94 \\
\hline $41-47$ & 43.5 & 1.567 & \pm 0.032 & $\begin{array}{l}+0.030 \\
-0.028\end{array}$ & $\begin{array}{l}+0.093 \\
-0.101\end{array}$ & 0.95 \\
\hline $47-55$ & 50.2 & 0.550 & \pm 0.017 & $\begin{array}{l}+0.018 \\
-0.018\end{array}$ & $\begin{array}{l}+0.037 \\
-0.039\end{array}$ & 0.95 \\
\hline $55-71$ & 60.3 & 0.1139 & \pm 0.0058 & $\begin{array}{l}+0.0039 \\
-0.0034\end{array}$ & $\begin{array}{l}+0.0074 \\
-0.0081\end{array}$ & 0.93 \\
\hline $71-95$ & 77.5 & 0.0105 & \pm 0.0014 & $\begin{array}{l}+0.0006 \\
-0.0007\end{array}$ & $\begin{array}{l}+0.0009 \\
-0.0010\end{array}$ & 0.96 \\
\hline \multicolumn{7}{|l|}{ SIScone } \\
\hline $17-21$ & 18.7 & 278.01 & \pm 0.51 & $\begin{array}{l}+4.31 \\
-4.30\end{array}$ & $\begin{array}{l}+10.89 \\
-11.52\end{array}$ & 0.80 \\
\hline $21-25$ & 22.7 & 90.82 & \pm 0.28 & $\begin{array}{l}+1.22 \\
-1.21\end{array}$ & $\begin{array}{l}+3.96 \\
-4.27\end{array}$ & 0.81 \\
\hline $25-29$ & 26.7 & 35.27 & \pm 0.18 & $\begin{array}{l}+0.49 \\
-0.48\end{array}$ & $\begin{array}{l}+1.65 \\
-1.75\end{array}$ & 0.82 \\
\hline $29-35$ & 31.4 & 13.059 & \pm 0.090 & $\begin{array}{l}+0.185 \\
-0.179\end{array}$ & $\begin{array}{l}+0.651 \\
-0.695\end{array}$ & 0.84 \\
\hline $35-41$ & 37.5 & 4.330 & \pm 0.051 & $\begin{array}{l}+0.108 \\
-0.107\end{array}$ & $\begin{array}{l}+0.233 \\
-0.252\end{array}$ & 0.85 \\
\hline $41-47$ & 43.6 & 1.639 & \pm 0.031 & $\begin{array}{l}+0.031 \\
-0.029\end{array}$ & $\begin{array}{l}+0.090 \\
-0.100\end{array}$ & 0.86 \\
\hline $47-55$ & 50.3 & 0.565 & \pm 0.016 & $\begin{array}{l}+0.020 \\
-0.020\end{array}$ & $\begin{array}{l}+0.034 \\
-0.039\end{array}$ & 0.87 \\
\hline $55-71$ & 60.5 & 0.1199 & \pm 0.0055 & $\begin{array}{l}+0.0045 \\
-0.0042\end{array}$ & $\begin{array}{l}+0.0085 \\
-0.0085\end{array}$ & 0.86 \\
\hline $71-95$ & 77.7 & 0.0108 & \pm 0.0013 & $\begin{array}{l}+0.0008 \\
-0.0008\end{array}$ & $\begin{array}{l}+0.0012 \\
-0.0013\end{array}$ & 0.90 \\
\hline
\end{tabular}

from the anti-k $k_{T}$ and SIScone measurements are consistent with those obtained from the $k_{T}$ analysis and have a similar precision.

\section{Summary and conclusions}

Measurements of differential cross sections for inclusive-jet photoproduction at a centre-ofmass energy of $318 \mathrm{GeV}$ using an integrated luminosity of $300 \mathrm{pb}^{-1}$ collected by the ZEUS detector have been presented. The cross sections refer to jets of hadrons of $E_{T}^{\mathrm{jet}}>17 \mathrm{GeV}$ and $-1<\eta^{\text {jet }}<2.5$ identified in the laboratory frame with the $k_{T}$, anti- $k_{T}$ or SIScone jet algorithms with jet radius $R=1$. The cross sections are given in the kinematic region of $Q^{2}<1 \mathrm{GeV}^{2}$ and $142<W_{\gamma p}<293 \mathrm{GeV}$. 


\section{RAPID COMMUNICATION}

Table 7

The measured differential cross sections $d \sigma / d \eta^{\text {jet }}$ based on different jet algorithms for inclusive-jet photoproduction with $E_{T}^{\text {jet }}>17 \mathrm{GeV}$ in the kinematic region given by $Q^{2}<1 \mathrm{GeV}^{2}$ and $142<W_{\gamma p}<293 \mathrm{GeV}$. Other details as in the caption to Table 2 .

\begin{tabular}{|c|c|c|c|c|c|c|}
\hline$\eta^{\text {jet }}$ bin & $\left\langle\eta^{\text {jet }}\right\rangle$ & $d \sigma / d \eta^{\text {jet }}(\mathrm{pb})$ & $\delta_{\text {stat }}$ & $\delta_{\text {syst }}$ & $\delta_{\mathrm{ES}}$ & $C_{\text {had }}$ \\
\hline \multicolumn{7}{|l|}{ anti- $k_{T}$} \\
\hline-0.75 to -0.50 & -0.58 & 65.63 & \pm 0.84 & $\begin{array}{l}+4.16 \\
-3.88\end{array}$ & $\begin{array}{l}+5.83 \\
-6.51\end{array}$ & 0.74 \\
\hline-0.50 to -0.25 & -0.35 & 200.3 & \pm 1.6 & $\begin{array}{l}+8.1 \\
-7.9\end{array}$ & $\begin{array}{l}+13.3 \\
-14.1\end{array}$ & 0.87 \\
\hline-0.25 to +0.00 & -0.12 & 369.5 & \pm 2.1 & $\begin{array}{l}+10.1 \\
-9.9\end{array}$ & $\begin{array}{l}+20.3 \\
-21.4\end{array}$ & 0.92 \\
\hline+0.00 to +0.25 & +0.13 & 518.6 & \pm 2.5 & $\begin{array}{l}+7.5 \\
-7.3\end{array}$ & $\begin{array}{l}+25.5 \\
-26.3\end{array}$ & 0.93 \\
\hline+0.25 to +0.50 & +0.38 & 634.6 & \pm 2.8 & $\begin{array}{l}+6.9 \\
-6.6\end{array}$ & $\begin{array}{l}+28.3 \\
-30.3\end{array}$ & 0.93 \\
\hline+0.50 to +0.75 & +0.63 & 722.2 & \pm 3.0 & $\begin{array}{l}+9.6 \\
-9.5\end{array}$ & $\begin{array}{l}+30.8 \\
-32.7\end{array}$ & 0.93 \\
\hline+0.75 to +1.00 & +0.87 & 748.2 & \pm 3.1 & $\begin{array}{l}+15.5 \\
-15.5\end{array}$ & $\begin{array}{l}+30.0 \\
-32.1\end{array}$ & 0.94 \\
\hline+1.00 to +1.25 & +1.12 & 732.3 & \pm 3.2 & $\begin{array}{l}+18.3 \\
-18.3\end{array}$ & $\begin{array}{l}+28.4 \\
-29.8\end{array}$ & 0.95 \\
\hline+1.25 to +1.50 & +1.38 & 649.7 & \pm 2.8 & $\begin{array}{l}+19.1 \\
-19.1\end{array}$ & $\begin{array}{l}+24.0 \\
-25.8\end{array}$ & 0.94 \\
\hline+1.50 to +1.75 & +1.63 & 610.9 & \pm 2.7 & $\begin{array}{l}+20.1 \\
-20.2\end{array}$ & $\begin{array}{l}+23.4 \\
-25.0\end{array}$ & 0.94 \\
\hline+1.75 to +2.00 & +1.87 & 569.5 & \pm 2.6 & $\begin{array}{l}+16.2 \\
-16.2\end{array}$ & $\begin{array}{l}+22.4 \\
-24.1\end{array}$ & 0.94 \\
\hline+2.00 to +2.25 & +2.13 & 536.2 & \pm 2.6 & $\begin{array}{l}+16.1 \\
-16.1\end{array}$ & $\begin{array}{l}+21.3 \\
-22.8\end{array}$ & 0.94 \\
\hline+2.25 to +2.50 & +2.38 & 488.6 & \pm 2.5 & $\begin{array}{l}+18.5 \\
-18.5\end{array}$ & $\begin{array}{l}+18.7 \\
-21.2\end{array}$ & 0.94 \\
\hline \multicolumn{7}{|l|}{ SIScone } \\
\hline-0.75 to -0.50 & -0.58 & 69.65 & \pm 0.87 & $\begin{array}{l}+4.67 \\
-4.36\end{array}$ & $\begin{array}{l}+6.21 \\
-7.06\end{array}$ & 0.66 \\
\hline-0.50 to -0.25 & -0.35 & 205.3 & \pm 1.6 & $\begin{array}{l}+8.7 \\
-8.7\end{array}$ & $\begin{array}{l}+13.5 \\
-14.7\end{array}$ & 0.77 \\
\hline-0.25 to +0.00 & -0.12 & 379.3 & \pm 2.2 & $\begin{array}{l}+11.0 \\
-10.9\end{array}$ & $\begin{array}{l}+20.4 \\
-22.1\end{array}$ & 0.81 \\
\hline+0.00 to +0.25 & +0.13 & 527.7 & \pm 2.6 & $\begin{array}{l}+8.3 \\
-8.0\end{array}$ & $\begin{array}{l}+25.6 \\
-27.1\end{array}$ & 0.82 \\
\hline+0.25 to +0.50 & +0.38 & 645.1 & \pm 2.9 & $\begin{array}{l}+6.8 \\
-6.7\end{array}$ & $\begin{array}{l}+28.1 \\
-30.6\end{array}$ & 0.82 \\
\hline+0.50 to +0.75 & +0.63 & 731.6 & \pm 3.1 & $\begin{array}{l}+9.1 \\
-9.0\end{array}$ & $\begin{array}{l}+30.1 \\
-32.5\end{array}$ & 0.82 \\
\hline+0.75 to +1.00 & +0.87 & 756.8 & \pm 3.2 & $\begin{array}{l}+13.8 \\
-13.8\end{array}$ & $\begin{array}{l}+29.9 \\
-31.8\end{array}$ & 0.83 \\
\hline+1.00 to +1.25 & +1.12 & 735.0 & \pm 3.3 & $\begin{array}{l}+14.7 \\
-14.6\end{array}$ & $\begin{array}{l}+27.7 \\
-29.3\end{array}$ & 0.83 \\
\hline+1.25 to +1.50 & +1.38 & 650.0 & \pm 2.9 & $\begin{array}{l}+15.2 \\
-15.3\end{array}$ & $\begin{array}{l}+23.3 \\
-24.3\end{array}$ & 0.82 \\
\hline+1.50 to +1.75 & +1.63 & 598.7 & \pm 2.7 & $\begin{array}{l}+14.3 \\
-14.3\end{array}$ & $\begin{array}{l}+22.3 \\
-23.1\end{array}$ & 0.81 \\
\hline+1.75 to +2.00 & +1.87 & 549.0 & \pm 2.6 & $\begin{array}{l}+10.8 \\
-10.9\end{array}$ & $\begin{array}{l}+20.9 \\
-22.2\end{array}$ & 0.81 \\
\hline+2.00 to +2.25 & +2.13 & 529.8 & \pm 2.5 & $\begin{array}{l}+14.1 \\
-14.1\end{array}$ & $\begin{array}{l}+20.5 \\
-22.0\end{array}$ & 0.80 \\
\hline+2.25 to +2.50 & +2.38 & 518.5 & \pm 2.5 & $\begin{array}{l}+24.3 \\
-24.4\end{array}$ & $\begin{array}{l}+20.1 \\
-21.6\end{array}$ & 0.79 \\
\hline
\end{tabular}




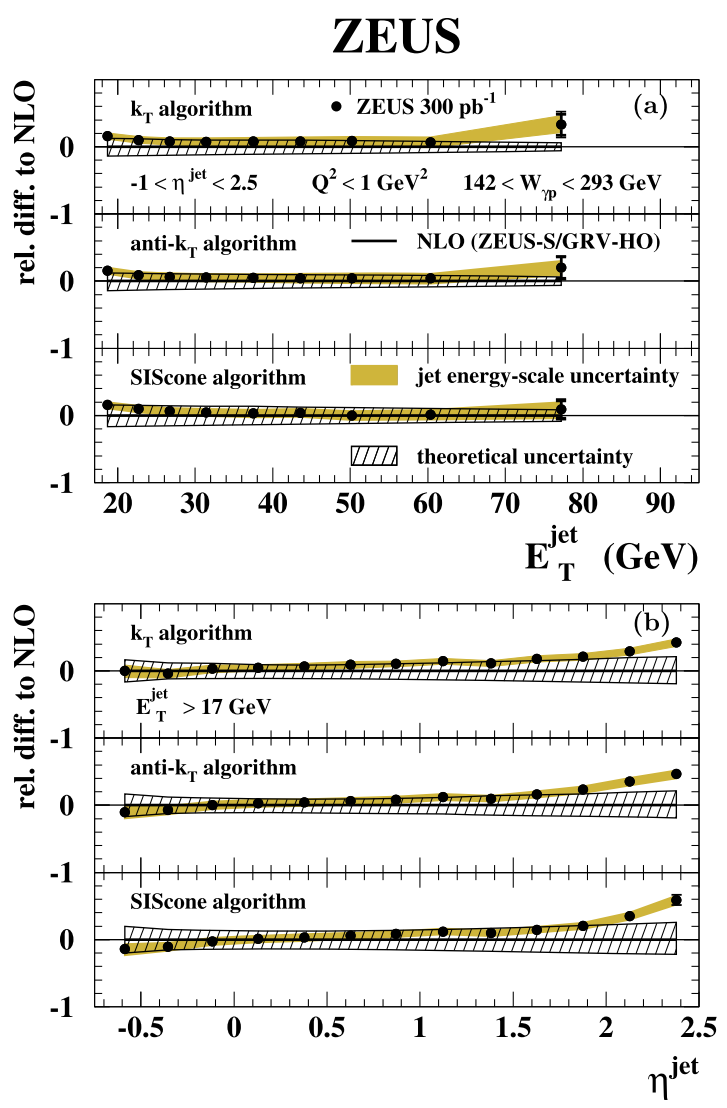

Fig. 14. The ratios between the measured cross sections (a) $d \sigma / d E_{T}^{\text {jet }}$ and (b) $d \sigma / d \eta^{\text {jet }}$ and the NLO QCD calculations (dots) from Fig. 13. Other details as in the caption to Fig. 2.

Measurements of single-differential cross sections were presented as functions of $E_{T}^{\mathrm{jet}}$ and $\eta^{\text {jet }}$. The NLO QCD calculations provide a good description of the measured cross sections, except at high $\eta^{\text {jet }}$. Non-perturbative effects not related to hadronisation and the influence of the photon PDFs were found to be most significant in this region.

Cross-section measurements were performed as functions of $E_{T}^{\mathrm{jet}}$ in different regions of $\eta^{\text {jet }}$. The data are well described by the NLO QCD predictions, except for jets with low $E_{T}^{\text {jet }}$ and high $\eta^{\text {jet }}$. These cross sections are sensitive to the parton densities in the proton and the photon in regions of phase space where the theoretical uncertainties are small. The precision measurements presented here are therefore of particular relevance for improving the determination of the PDFs in future QCD fits.

A detailed comparison between the measurements for the three jet algorithms was performed. The measured cross sections for the three jet algorithms have a similar shape, normalisation and precision. The NLO QCD calculations of inclusive-jet cross sections and their uncertainties for the different jet algorithms were also compared: the predictions describe the data well, except at high $\eta^{\text {jet}}$; the calculations based on the SIScone algorithm are somewhat less precise than those based on the $k_{T}$ or anti- $k_{T}$ due to a larger contribution from terms beyond NLO. The ratios 


\section{RAPID COMMUNICATION}

Table 8

Experimental, theoretical and total uncertainties in the determination of $\alpha_{S}\left(M_{Z}\right)$ from the $k_{T}$, anti-k $k_{T}$ and SIScone analyses.

\begin{tabular}{llll}
\hline & $k_{T}$ & anti- $k_{T}$ & SIScone \\
\hline Experimental uncertainties & & & \\
jet energy scale & $+1.8 \%$ & $+1.8 \%$ & $+1.7 \%$ \\
luminosity & $-1.7 \%$ & $\pm 0.6 \%$ & $\pm 0.6 \%$ \\
uncorrelated & $\pm 0.6 \%$ & $+0.3 \%$ & $+0.3 \%$ \\
statistical & $+0.3 \%$ & $-0.4 \%$ \\
Theoretical uncertainties & $-0.4 \%$ & $\pm 0.2 \%$ & $\pm 0.2 \%$ \\
terms beyond NLO & $\pm 0.2 \%$ & & $+3.2 \%$ \\
photon PDFs & & $+2.3 \%$ & $-3.3 \%$ \\
proton PDFs & $+2.4 \%$ & $-2.4 \%$ & $+1.9 \%$ \\
hadronisation & $-2.5 \%$ & $+2.2 \%$ & $\pm 1.0 \%$ \\
Total uncertainty & $+2.3 \%$ & $\pm 1.0 \%$ & $\pm 0.2 \%$ \\
& $-0.9 \%$ & $\pm 0.4 \%$ & \\
\hline
\end{tabular}

Table 9

The $\alpha_{s}$ values determined in each $\left\langle E_{T}^{\mathrm{jet}}\right\rangle$ value from the analysis of the measured $d \sigma / d E_{T}^{\mathrm{jet}}$ cross section based on the $k_{T}$ jet algorithm. The uncorrelated $\left(\delta_{\text {uncorr }}\right)$ and correlated $\left(\delta_{\text {corr }}\right)$ experimental and theoretical $\left(\delta_{\text {th }}\right)$ uncertainties are listed separately.

\begin{tabular}{|c|c|c|c|c|}
\hline$\left\langle E_{T}^{\mathrm{jet}}\right\rangle(\mathrm{GeV})$ & $\alpha_{s}$ & $\delta_{\text {uncorr }}$ & $\delta_{\text {corr }}$ & $\delta_{\text {th }}$ \\
\hline 22.7 & 0.1561 & \pm 0.0011 & $\begin{array}{l}+0.0035 \\
-0.0048\end{array}$ & $\begin{array}{l}+0.0106 \\
-0.0089\end{array}$ \\
\hline 26.7 & 0.1493 & \pm 0.0007 & $\begin{array}{l}+0.0033 \\
-0.0034\end{array}$ & $\begin{array}{l}+0.0083 \\
-0.0070\end{array}$ \\
\hline 31.4 & 0.1443 & \pm 0.0005 & $\begin{array}{l}+0.0035 \\
-0.0030\end{array}$ & $\begin{array}{l}+0.0069 \\
-0.0059\end{array}$ \\
\hline 37.5 & 0.1396 & \pm 0.0007 & $\begin{array}{l}+0.0032 \\
-0.0031\end{array}$ & $\begin{array}{l}+0.0057 \\
-0.0051\end{array}$ \\
\hline 43.6 & 0.1359 & \pm 0.0011 & $\begin{array}{l}+0.0032 \\
-0.0030\end{array}$ & $\begin{array}{l}+0.0051 \\
-0.0047\end{array}$ \\
\hline 50.2 & 0.1328 & \pm 0.0014 & $\begin{array}{l}+0.0037 \\
-0.0034\end{array}$ & $\begin{array}{l}+0.0047 \\
-0.0045\end{array}$ \\
\hline 60.3 & 0.1283 & \pm 0.0024 & $\begin{array}{l}+0.0040 \\
-0.0036\end{array}$ & $\begin{array}{l}+0.0041 \\
-0.0041\end{array}$ \\
\hline
\end{tabular}

of the cross sections based on the different jet algorithms were also presented. The measured ratios are well reproduced by the $\mathcal{O}\left(\alpha_{s}^{2}\right)$ predictions, demonstrating the ability of the pQCD calculations including up to three partons in the final state to account adequately for the details of the differences between the SIScone and the $k_{T}$ or anti- $k_{T}$ jet algorithms.

The measured cross sections were used to determine values of $\alpha_{s}\left(M_{Z}\right)$. QCD fits to the cross section $d \sigma / d E_{T}^{\text {jet }}$ for $21<E_{T}^{\text {jet }}<71 \mathrm{GeV}$ based on the $k_{T}$ jet algorithm yielded

$$
\alpha_{S}\left(M_{Z}\right)=0.1206_{-0.0022}^{+0.0023} \text { (exp.) }{ }_{-0.0035}^{+0.0042} \text { (th.). }
$$




\section{ZEUS}
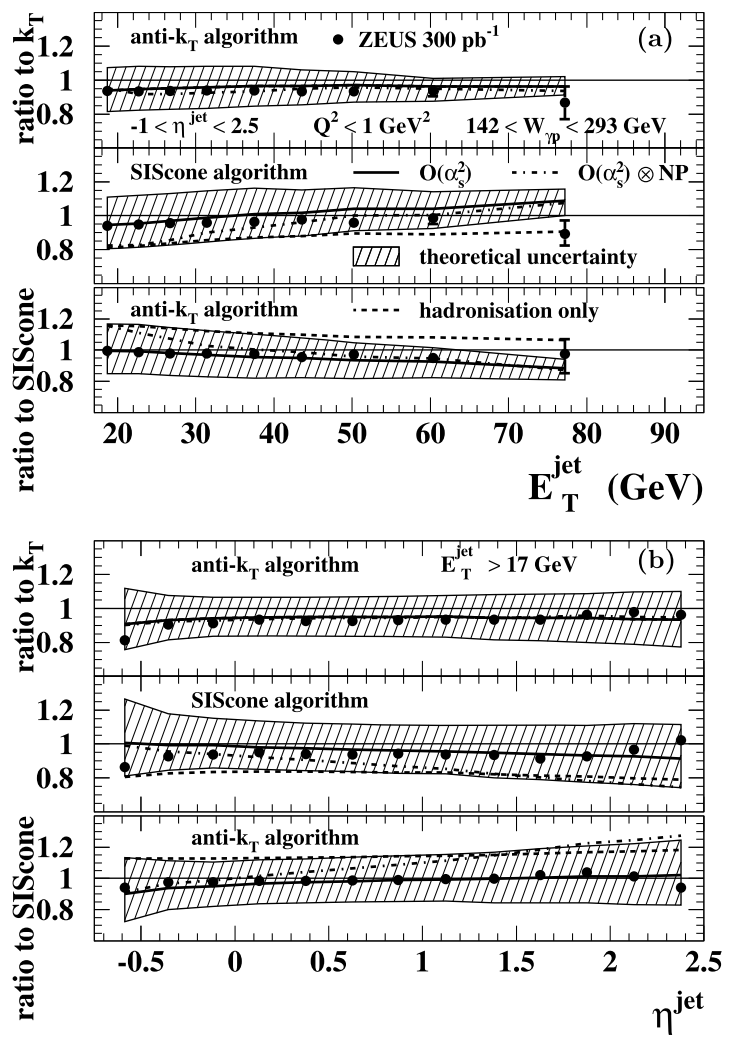

Fig. 15. The ratios of the measured cross sections anti- $k_{T} / k_{T}$, SIScone $/ k_{T}$ and anti- $k_{T} /$ SIScone (dots) as functions of (a) $E_{T}^{\text {jet }}$ and (b) $\eta^{\text {jet }}$. In these plots, the outer error bars also include the uncertainty on the absolute energy scale of the jets. The predicted ratios based on calculations which include up to $\mathcal{O}\left(\alpha_{s}^{2}\right)$ terms are also shown (solid lines). The hatched bands display the theoretical uncertainty on the ratio. The dashed lines indicate the ratios of the hadronisation correction factors and the dash-dotted lines represent the ratios of the NLO QCD calculations including an estimation of non-perturbative effects (see text). Other details as in the caption to Fig. 2.

This value is in good agreement with the world and HERA averages. The extracted values of $\alpha_{s}$ as a function of $E_{T}^{\text {jet }}$ are in good agreement with the predicted running of the strong coupling over a large range in $E_{T}^{\text {jet }}$.

\section{Acknowledgements}

We thank the DESY Directorate for their strong support and encouragement. The remarkable achievements of the HERA machine group were essential for the successful completion of this work and are greatly appreciated. We are grateful for the support of the DESY computing and network services. The design, construction, installation and running of the ZEUS detector were made possible owing to the ingenuity and effort of many people who are not listed as authors. 


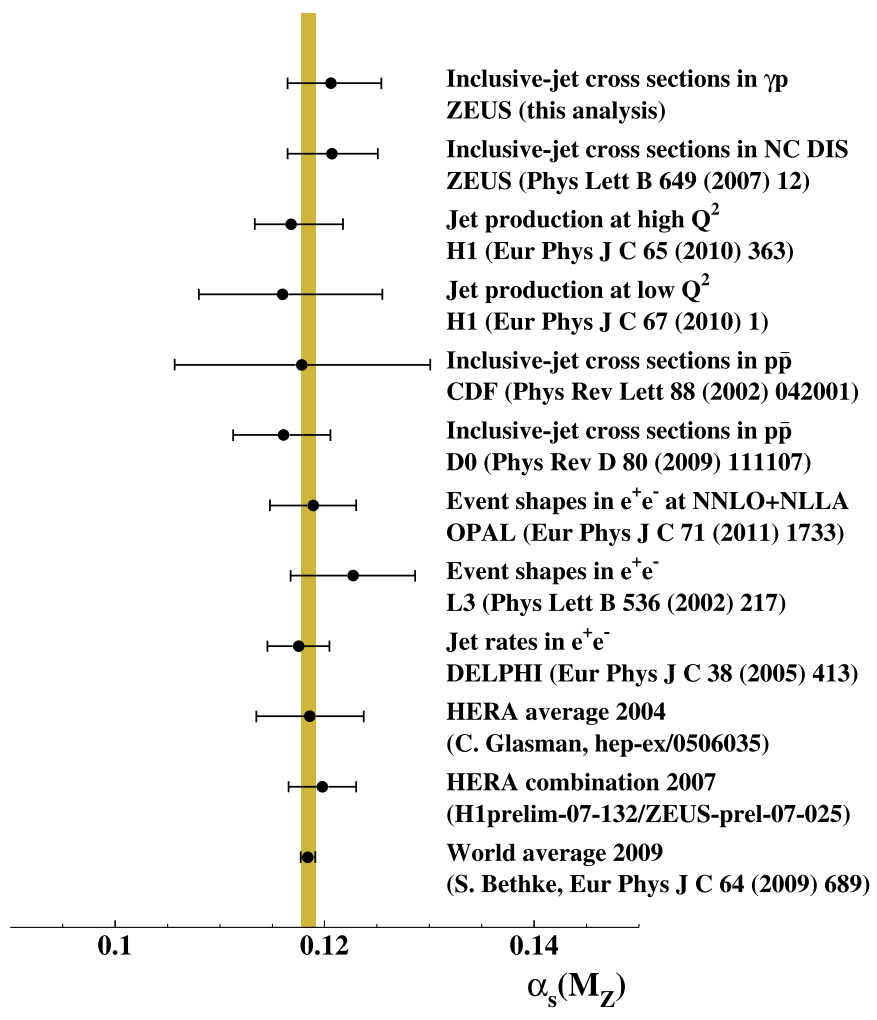

Fig. 16. Extracted $\alpha_{S}\left(M_{Z}\right)$ value from this analysis (upper dot). For comparison, determinations from other experiments and reactions, the HERA average 2004, the HERA combination 2007 and the world average 2009 are also shown. The horizontal error bars represent the experimental and theoretical uncertainties added in quadrature. The shaded band represents the uncertainty of the world average.

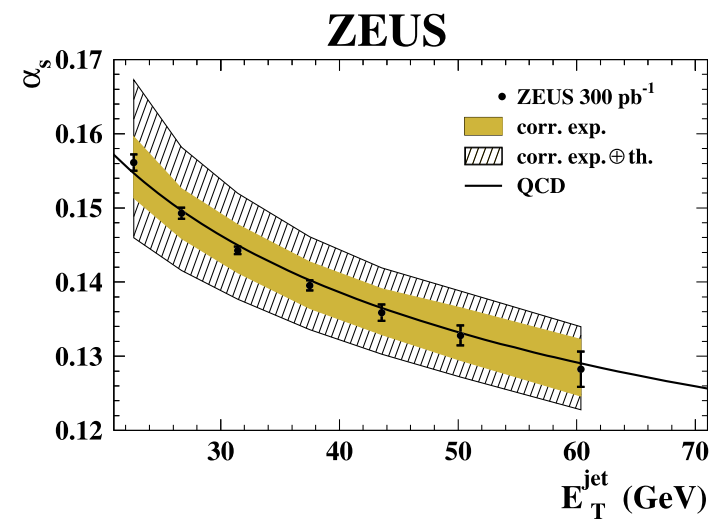

Fig. 17. The $\alpha_{s}$ values determined in each $\left\langle E_{T}^{\text {jet }}\right\rangle$ value from the analysis of the measured $d \sigma / d E_{T}^{\text {jet }}$ cross section based on the $k_{T}$ jet algorithm (dots). The error bars represent the uncorrelated experimental uncertainties; the shaded area represents the correlated experimental uncertainties and the hatched area represents the correlated experimental and theoretical uncertainties added in quadrature. The solid line indicates the renormalisation-group prediction at two loops obtained from the corresponding $\alpha_{S}\left(M_{Z}\right)$ value determined in this analysis. 


\section{References}

[1] H1 Collaboration, C. Adloff, et al., Eur. Phys. J. C 19 (2001) 289.

[2] ZEUS Collaboration, S. Chekanov, et al., Phys. Lett. B 547 (2002) 164.

[3] ZEUS Collaboration, S. Chekanov, et al., Phys. Lett. B 551 (2003) 226.

[4] ZEUS Collaboration, S. Chekanov, et al., Nucl. Phys. B 765 (2007) 1.

[5] ZEUS Collaboration, S. Chekanov, et al., Phys. Lett. B 649 (2007) 12.

[6] H1 Collaboration, F.D. Aaron, et al., Eur. Phys. J. C 65 (2010) 363.

[7] H1 Collaboration, F.D. Aaron, et al., Eur. Phys. J. C 67 (2010) 1.

[8] ZEUS Collaboration, J. Breitweg, et al., Phys. Lett. B 507 (2001) 70.

[9] ZEUS Collaboration, S. Chekanov, et al., Eur. Phys. J. C 23 (2002) 13.

[10] H1 Collaboration, C. Adloff, et al., Phys. Lett. B 515 (2001) 17.

[11] ZEUS Collaboration, S. Chekanov, et al., Eur. Phys. J. C 44 (2005) 183.

[12] ZEUS Collaboration, H. Abramowicz, et al., Phys. Rev. D 85 (2012) 052008.

[13] ZEUS Collaboration, S. Chekanov, et al., Phys. Lett. B 560 (2003) 7.

[14] H1 Collaboration, C. Adloff, et al., Eur. Phys. J. C 29 (2003) 497.

[15] ZEUS Collaboration, J. Breitweg, et al., Eur. Phys. J. C 11 (1999) 35.

[16] ZEUS Collaboration, S. Chekanov, et al., Eur. Phys. J. C 23 (2002) 615.

[17] ZEUS Collaboration, S. Chekanov, et al., Phys. Lett. B 531 (2002) 9.

[18] H1 Collaboration, C. Adloff, et al., Eur. Phys. J. C 25 (2002) 13.

[19] H1 Collaboration, A. Aktas, et al., Phys. Lett. B 639 (2006) 21.

[20] ZEUS Collaboration, S. Chekanov, et al., Phys. Rev. D 76 (2007) 072011.

[21] ZEUS Collaboration, J. Breitweg, et al., Phys. Lett. B 443 (1998) 394.

[22] ZEUS Collaboration, S. Chekanov, et al., Nucl. Phys. B 792 (2008) 1.

[23] H1 Collaboration, C. Adloff, et al., Nucl. Phys. B 545 (1999) 3.

[24] ZEUS Collaboration, S. Chekanov, et al., Phys. Lett. B 558 (2003) 41.

[25] ZEUS Collaboration, S. Chekanov, et al., Nucl. Phys. B 700 (2004) 3.

[26] ZEUS Collaboration, S. Chekanov, et al., Eur. Phys. J. C 31 (2003) 149.

[27] ZEUS Collaboration, S. Chekanov, et al., Phys. Rev. D 78 (2008) 032004.

[28] S. Catani, et al., Nucl. Phys. B 406 (1993) 187.

[29] S.D. Ellis, D.E. Soper, Phys. Rev. D 48 (1993) 3160.

[30] ZEUS Collaboration, S. Chekanov, et al., Eur. Phys. J. C 42 (2005) 1.

[31] M. Cacciari, G.P. Salam, G. Soyez, JHEP 0804 (2008) 063.

[32] G.P. Salam, G. Soyez, JHEP 0705 (2007) 086.

[33] ZEUS Collaboration, H. Abramowicz, et al., Phys. Lett. B 691 (2010) 127.

[34] C.H. Llewellyn Smith, Phys. Lett. B 79 (1978) 83;

I. Kang, C.H. Llewellyn Smith, Nucl. Phys. B 166 (1980) 413;

J.F. Owens, Phys. Rev. D 21 (1980) 54;

M. Fontannaz, A. Mantrach, D. Schiff, Z. Phys. C 6 (1980) 241.

[35] W.J. Stirling, Z. Kunszt, in: R.D. Peccei (Ed.), Proc. HERA Workshop, vol. 2, DESY, Hamburg, Germany, 1987, p. 331;

M. Drees, F. Halzen, Phys. Rev. Lett. 61 (1988) 275;

M. Drees, R.M. Godbole, Phys. Rev. Lett. 61 (1988) 682;

M. Drees, R.M. Godbole, Phys. Rev. D 39 (1989) 169;

H. Baer, J. Ohnemus, J.F. Owens, Z. Phys. C 42 (1989) 657;

H. Baer, J. Ohnemus, J.F. Owens, Phys. Rev. D 40 (1989) 2844.

[36] ZEUS Collaboration, M. Derrick, et al., Phys. Lett. B 293 (1992) 465.

[37] ZEUS Collaboration, U. Holm (Ed.), The ZEUS Detector. Status Report, DESY, 1993 (unpublished), available on http://www-zeus.desy.de/bluebook/bluebook.html.

[38] N. Harnew, et al., Nucl. Instr. Meth. A 279 (1989) 290;

B. Foster, et al., Nucl. Phys. B Proc. Suppl. 32 (1993) 181;

B. Foster, et al., Nucl. Instr. Meth. A 338 (1994) 254. 
[39] A. Polini, et al., Nucl. Instr. Meth. A 581 (2007) 656.

[40] M. Derrick, et al., Nucl. Instr. Meth. A 309 (1991) 77;

A. Andresen, et al., Nucl. Instr. Meth. A 309 (1991) 101;

A. Caldwell, et al., Nucl. Instr. Meth. A 321 (1992) 356;

A. Bernstein, et al., Nucl. Instr. Meth. A 336 (1993) 23.

[41] J. Andruszków, et al., DESY, 1992, Preprint DESY-92-066; ZEUS Collaboration, M. Derrick, et al., Z. Phys. C 63 (1994) 391; J. Andruszków, et al., Acta Phys. Polon. B 32 (2001) 2025.

[42] M. Helbich, et al., Nucl. Instr. Meth. A 565 (2006) 572.

[43] W.H. Smith, K. Tokushuku, L.W. Wiggers, in: C. Verkerk, W. Wojcik (Eds.), Proc. Computing in High-Energy Physics (CHEP), Annecy, France, Sept. 1992, CERN, Geneva, Switzerland, 1992, p. 222; also preprint DESY $92-$ 150B.

[44] S.M. Berman, J.D. Bjorken, J.B. Kogut, Phys. Rev. D 4 (1971) 3388.

[45] J.E. Huth, et al., Research directions for the decade, in: E.L. Berger (Ed.), Proc. of Summer Study on High Energy Physics, 1990, World Scientific, 1992, p. 134; also preprint FERMILAB-CONF-90-249-E.

[46] M. Cacciari, G.P. Salam, Phys. Lett. B 641 (2006) 57.

[47] T. Sjöstrand, Comput. Phys. Comm. 82 (1994) 74.

[48] G. Marchesini, et al., Comput. Phys. Comm. 67 (1992) 465;

G. Corcella, et al., JHEP 0101 (2001) 010.

[49] B. Andersson, et al., Phys. Rep. 97 (1983) 31.

[50] T. Sjöstrand, Comput. Phys. Comm. 39 (1986) 347;

T. Sjöstrand, M. Bengtsson, Comput. Phys. Comm. 43 (1987) 367.

[51] B.R. Webber, Nucl. Phys. B 238 (1984) 492.

[52] H.L. Lai, et al., Phys. Rev. D 55 (1997) 1280.

[53] M. Glück, E. Reya, A. Vogt, Phys. Rev. D 45 (1992) 3986;

M. Glück, E. Reya, A. Vogt, Phys. Rev. D 46 (1992) 1973.

[54] T. Sjöstrand, M. van Zijl, Phys. Rev. D 36 (1987) 2019.

[55] R. Brun, et al., GEANT3, Technical Report CERN-DD/EE/84-1, CERN, 1987.

[56] M. Wing (on behalf of the ZEUS Collaboration), in: Proc. of the 10th International Conference on Calorimetry in High Energy Physics, hep-ex/0206036, 2002.

[57] A.D. Martin, et al., Eur. Phys. J. C 4 (1998) 463.

[58] P. Aurenche, J.-P. Guillet, M. Fontannaz, Z. Phys. C 64 (1994) 621.

[59] M. Klasen, T. Kleinwort, G. Kramer, Eur. Phys. J. C 1 (1998) 1.

[60] G. Kramer, Theory of Jets in Electron-Positron Annihilation, Springer, Berlin, 1984.

[61] ZEUS Collaboration, S. Chekanov, et al., Phys. Rev. D 67 (2003) 012007.

[62] M.R. Whalley, D. Bourilkov, R.C. Group, hep-ph/0508110, 2005.

[63] S. Bethke, J. Phys. G 26 (2000) R27; Updated in S. Bethke, Eur. Phys. J. C 64 (2009) 689.

[64] P. Aurenche, M. Fontannaz, J.Ph. Guillet, Eur. Phys. J. C 44 (2005) 395.

[65] F. Cornet, P. Jankowski, M. Krawczyk, Phys. Rev. D 70 (2004) 093004.

[66] A.D. Martin, et al., Eur. Phys. J. C 63 (2009) 189.

[67] H1 Collaboration, ZEUS Collaboration, F.D. Aaron, et al., JHEP 1001 (2010) 109.

[68] R.W.L. Jones, et al., JHEP 0312 (2003) 007.

[69] J. Pumplin, et al., JHEP 0207 (2002) 012; D. Stump, et al., JHEP 0310 (2003) 046.

[70] CDF Collaboration, T. Affolder, et al., Phys. Rev. Lett. 88 (2002) 042001; DØ Collaboration, V.M. Abazov, et al., Phys. Rev. D 80 (2009) 111107.

[71] L3 Collaboration, P. Achard, et al., Phys. Lett. B 536 (2002) 217; DELPHI Collaboration, J. Abdallah, et al., Eur. Phys. J. C 38 (2005) 413; OPAL Collaboration, G. Abbiendi, et al., Eur. Phys. J. C 71 (2011) 1733.

[72] C. Glasman, in: S.R. Dasu, W.H. Smith (Eds.), Proc. of the 13th International Workshop on Deep Inelastic Scattering, Madison, USA, p. 689; also preprint hep-ex/0506035, 2005. 


\section{RAPID COMMUNICATION}

[73] C. Glasman, in: Proc. of the HEP2007 International Europhysics Conference on High Energy Physics, J. Phys. Conf. Ser. 110 (2008) 022013; also preprint arXiv:0709.4426.

[74] D.J. Gross, F. Wilczek, Phys. Rev. Lett. 30 (1973) 1343;

H.D. Politzer, Phys. Rev. Lett. 30 (1973) 1346;

D.J. Gross, F. Wilczek, Phys. Rev. D 8 (1973) 3633;

H.D. Politzer, Phys. Rep. 14 (1974) 129. 